\title{
ON THE STRUCTURE OF LOCAL COHOMOLOGY MODULES FOR MONOMIAL CURVES IN $P_{K}^{3}$
}

\author{
H. BRESINSKY, F. CURTIS, M. FIORENTINI AND L. T. HOA
}

\section{Introduction}

Our setting for this paper is projective 3 -space $P_{K}^{3}$ over an algebraically closed field $K$. By a curve $C \subset P_{K}^{3}$ is meant a 1 -dimensional, equidimensional projective algebraic set, which is locally Cohen-Macaulay. Let $M(C)=\bigoplus_{n \in \mathbf{Z}} H^{1}\left(P_{K}^{3}\right.$, $\mathscr{I}_{C}(n)$ ) be the Hartshorne-Rao module of finite length (cf. [R]). Here $\mathbf{Z}$ is the set of integers and $\mathscr{I}_{C}$ the ideal sheaf of $C$. In [GMV] it is shown that $M(C) \cong H_{\underline{m}}^{1}(\bar{R})$, where $\bar{R}=R / I(C)=K\left[x_{0}, \ldots, x_{3}\right] / I(C), I(C)$ is the homogeneous ideal of $C$, $\underline{m}=\left(x_{0}, \ldots, x_{3}\right) R$ and $H_{\underline{m}}^{1}(M)$ is the first local cohomology module of the $R$-module $M$ with respect to $m$. Thus there exists a smallest nonnegative integer $k$ $\in \mathbf{N}$ such that $\underline{m}^{k} H_{\underline{m}}^{1}(\bar{R})=0$, (see also the discussion on the 1 -st local cohomology module in [GW]). Also in [GMV] it is shown that $k=0$ if and only if $C$ is arithmetically Cohen-Macaulay and $C$ is arithmetically Buchsbaum if and only if $k$ $\leq 1$. We therefore have the following natural definition.

Definition 1.1. For a curve $C \subseteq P_{K}^{3}, C$ is said to be strictly $k$-Buchsbaum if $k$ is minimal in $\mathbf{N}$ such that $\underline{m}^{k} H_{\underline{m}}^{1}(\bar{R})=0 . C$ is said to be $k$-Buchsbaum if $\underline{m}^{k} H_{\underline{m}}^{1}(\bar{R})=0$.

If $C$ is strictly $k$-Buchsbaum, then we set $k=k(C)$ and call $k(C)$ the Buchsbaum number of $C$.

It is our purpose in this paper to investigate for the class of monomial curves $C\left(n_{1}, n_{2}, n_{3}\right) \subset P_{K}^{3}$ the integer $k\left(C\left(n_{1}, n_{2}, n_{3}\right)\right)$. These curves are defined by their generic zero $\left(s^{n_{3}}, s^{n_{3}-n_{1}} t^{n_{1}}, s^{n_{3}-n_{2}} t^{n_{2}}, t^{n_{3}}\right)$, where $n_{1}<n_{2}<n_{3}$ are positive integers and g.c.d. $\left(n_{1}, n_{2}, n_{3}\right)=1$. For some of these curves $k\left(C\left(n_{1}, n_{2}, n_{3}\right)\right)$ was obtained in $[\mathrm{FH}],[\mathrm{H}]$ and $[\mathrm{FV}]$ and we will discuss some of these results as consequences of out own investigations (see also [HV] and [MM]).

Our own main result is that $k(C)=\operatorname{diam}(M(C))$ for all monomial curves in

Received April 26, 1993. 
$P_{K}^{3}$. Here $\operatorname{diam}(M)$, the diameter of a $\mathbf{Z}$-graded module $M$ of finite length, is explained as follows. Let $[M]_{n}$ be the elements in $M$ of degree $n$, let $a(M)=$ $\min \left\{n ;[M]_{n} \neq 0\right\}, e(M)=\max \left\{n ;[M]_{n} \neq 0\right\}$. Then $\operatorname{diam}(M)=e(M)-a(M)$ +1 , which, since $M$ is of finite length, is an integer. For curves $C \subseteq P_{K}^{3}$, $H_{\underline{m}}^{1}(\bar{R})$ is a $\boldsymbol{Z}$-graded module of finite length and therefore always $k(C)$ $\leq \operatorname{diam}\left(H_{\underline{m}}^{1}(\bar{R})\right)$. Thus our result implies that for all monomial curves $C\left(n_{1}, n_{2}\right.$, $\left.n_{3}\right) \subseteq P_{K}^{3}, k\left(C\left(n_{1}, n_{2}, n_{3}\right)\right)$ is, in this sense, as large as possible. We prove this result in Section 2. We will use there mainly the language of semigroups to describe $H_{\underline{m}}^{1}(\bar{R})$. For this let $\mathbf{N}$ be the set of nonnegative integers, $n_{0}=0$ and $t_{i}=$ $\left(n_{3}-n_{i}, n_{i}\right) \in \mathrm{N}^{2}, 0 \leq i \leq 3$. Let $S \subseteq \mathrm{N}^{2}$ be the semigroup generated by $I=$ $\left\{t_{i} ; 0 \leq i \leq 3\right\}$ and $H=\left\{(\alpha, \beta) \in \mathbf{N}^{2} ; \alpha+\beta \equiv 0 \bmod n_{3}\right\}$. Let $S_{0}=\left\langle n_{3}-n_{2}\right.$, $\left.n_{3}-n_{1}, n_{3}\right\rangle=\left\{z ; z=\sum_{i=0}^{2} z_{i}\left(n_{3}-n_{i}\right), z_{i} \in \mathrm{N}\right\}, S_{1}=\left\langle n_{1}, n_{2}, n_{3}\right\rangle$. For $i \in\{0,1\}$ we define $A_{i}\left(S_{i}, n_{3}\right)=\left\{0, \omega_{i}(1), \ldots, \omega_{i}\left(n_{3}-1\right)\right\}, \omega_{i}(j)=\min \left\{z ; z \in S_{i}, z \equiv j\right.$ $\left.\bmod n_{3}\right\}$, to be the Apery sequence of $S_{i}$ with respect to $n_{3}$. We set $S^{\prime}=\{e \in H$; $e+m_{0} t_{0} \in S, e+m_{1} t_{3} \in S$, for some $\left.m_{0}, m_{1} \in \mathrm{N}\right\}$. By identifying $\bar{R}$ with the graded ring $K[S]$, we then have by $[\mathrm{B} 1],[\mathrm{TH}]$ and $[\mathrm{FH}]$ the following lemma.

LEMma 1.1.

(i) $H_{\underline{m}}^{1}(K[S])=K\left[S^{\prime} \backslash S\right]$, where $\underline{m}$ is identified with $K[S \backslash(0)]$.

(ii) $S \subseteq S^{\prime}=H \cap\left(S_{0} \times S_{1}\right)$.

(iii) $K[S]$ is a $k$-Buchsbaum ring if and only if one of the following equivalent conditions holds:

a) $S^{\prime}+k(S \backslash(0)) \subseteq S$.

b) For $J=H \cap\left(A_{0}\left(S_{0}, n_{3}\right) \times A_{1}\left(S_{1}, n_{3}\right)\right) \backslash S, J+k I \subseteq S$.

(In both a) and b) $k$ times a set means the set added to itself $k$ times.)

In Section 3 we relate our result in Section 2 to the algorithm in [BR], which obtains a minimal generating set $\mathscr{B}\left(n_{1}, n_{2}, n_{3}\right)$ of binomials for $I\left(C\left(n_{1}, n_{2}, n_{3}\right)\right)$. This enables us to calculate $k\left(C\left(n_{1}, n_{2}, n_{3}\right)\right)$ by considering a subset of $\mathscr{B}\left(n_{1}, n_{2}, n_{3}\right)$. For this we need to relate a minimal generating set of $H_{\underline{m}}^{1}(\bar{R})$ to $\mathscr{B}\left(n_{1}, n_{2}, n_{3}\right)$ (see Lemma 6 in [BSS]).

In Section 4 we show that the Castelnuovo-Mumford regularity for $\bar{R}=$ $R / I\left(C\left(n_{1}, n_{2}, n_{3}\right)\right), \operatorname{reg}(\bar{R})=e\left(H_{m}^{1}(\bar{R})\right)+1$ if $\bar{R}$ is not Cohen-Macaulay.

Section 5 establishes that for the subset of non Cohen-Macaulay monomial curves $C\left(1=n_{1}, n_{2}, n_{3}\right), k\left(C\left(n_{1}, n_{2}, n_{3}\right)\right)=n_{2}-2$.

We conclude our paper with Section 6 which deals with liaison among monomial curves in $P_{K}^{3}$. It is shown that no two curves $C\left(1, n_{2}, n_{3}\right)$, which are not arithmetically Cohen-Macaulay, are in the same even linkage class. Our main re- 
sult in this section is that for a fixed nonnegative integer $k$ there are only finitely many linkage classes among strictly $k$-Buchsbaum monomial curves in $P_{K}^{3}$.

Acknowledgement. This paper was written during the stay of the fourth named author at the Department of Mathematics, University of Ferrara, made possible by a grant of CNR. He would like to thank both institutions for their support.

\section{The equality of $k\left(C\left(n_{1}, n_{2}, n_{3}\right)\right)$ and $\operatorname{diam}\left(H_{\underline{m}}^{1}\left(R / I\left(C\left(n_{1}, n_{2}, n_{3}\right)\right)\right.\right.$}

We need to state some more definitions and prove some preliminary lemmas and propositions. We will use the notation and terminology introduced in 1 .

Definition 2.1. For $j \in S_{1}$ let the $S_{1}$-degree of $j$ be

$$
\delta_{1}(j)=\min \left\{a_{1}+a_{2}+a_{3} ; j=a_{1} n_{1}+a_{2} n_{2}+a_{3} n_{3}, a_{i} \in \mathbf{N}\right\} .
$$

Analogously we define the $S_{0}$-degree $\delta_{0}(i)$ for $i \in S_{0}$.

For $(i, j) \in H$, we define its degree to be the number $\delta(i, j)=(i+j) / n_{3} \geq 0$.

Definition 2.2. For $\left(i_{1}, j_{1}\right)$ and $\left(i_{2}, j_{2}\right)$ in $S^{\prime}$ we define the following partial orders:

(i ) $\left(i_{1}, j_{1}\right) \leq_{S}\left(i_{2}, j_{2}\right)$ if $\left(i_{2}-i_{1}, j_{2}-j_{1}\right) \in S$,

(ii) $\left(i_{1}, j_{1}\right) \leq_{S^{\prime}}\left(i_{2}, j_{2}\right)$ if $\left(i_{2}-i_{1}, j_{2}-j_{1}\right) \in S^{\prime}$.

$(i, j) \in S^{\prime} \backslash S$ is an $S$-maximal (minimal) element, if $(i, j)$ is maximal (minimal) in $S^{\prime} \backslash S$ with respect to the partial order $\leq_{s} .(i, j) \in S^{\prime} \backslash S$ is an $S^{\prime}$-minimal element, if $(i, j)$ is minimal in $S^{\prime} \backslash S$ with respect to the partial order $\leq_{S^{\prime}}$.

Lemma 2.1 ([B1], $[\mathrm{K}])$. Let $(i, j) \in S^{\prime}$. The following are equivalent:

(i) $(i, j) \in S$.

(ii) $\delta(i, j) \geq \delta_{1}(j)$.

(iii) $\delta(i, j) \geq \delta_{0}(i)$.

Proof. (i) $\Rightarrow$ (ii). Assume $(i, j) \in S,(i, j)=\sum_{i=0}^{3} a_{i} t_{i}$. Then $\delta(i, j)=\sum_{i=0}^{3} a_{i}$ $\geq a_{1}+a_{2}+a_{3} \geq \delta_{1}(j)$.

(ii) $\Rightarrow$ (i). Assume $\delta(i, j) \geq \delta_{1}(j)$, let $j=a_{1} n_{1}+a_{2} n_{2}+a_{3} n_{3}$ with $a_{1}+a_{2}+$ $a_{3}=\delta_{1}(j)$. Let $a_{0}=\delta(i, j)-\left(a_{1}+a_{2}+a_{3}\right)$ and $\left(i_{1}, j_{1}\right)=\sum_{i=0}^{3} a_{i} t_{i}$. Then $j_{1}=$ $j, \delta\left(i_{1}, j_{1}\right)=\delta(i, j)$, thus $i_{1}=i$ and $(i, j) \in S$.

(i) $\Leftrightarrow$ (iii) follows by symmetry as in the preceding. (Symmetry here and 
throughout means switching from one of $S_{0}$ or $S_{1}$ to the other.)

Remark 2.1. The following elementary facts will be used in the sequel.

a) If $\delta_{1}\left(a_{1} n_{1}+a_{2} n_{2}+a_{3} n_{3}\right)=a_{1}+a_{2}+a_{3}$ and $b_{i} \leq a_{i}, 1 \leq i \leq 3$, then $\delta_{1}\left(b_{1} n_{1}+b_{2} n_{2}+b_{3} n_{3}\right)=b_{1}+b_{2}+b_{3}$. Otherwise $\delta_{1}\left(a_{1} n_{1}+a_{2} n_{2}+a_{3} n_{3}\right) \neq a_{1}+$ $a_{2}+a_{3}$.

b) If $n_{1}<n_{2}<n_{3}$ are positive integers, then the following are true for nonnegative integers $a, b, c$ :

(i) If $a \neq 0$ and $a n_{1}=b n_{2}+c n_{3}$ then $a>b+c$,

(ii) If $c \neq 0$ and $c n_{3}=a n_{1}+b n_{2}$ then $c<a+b$.

Lemma 2.2. Let $(i, j) \in S^{\prime} \backslash(0)$. The following are equivalent:

(i) $(i, j)$ is an $S$-minimal element of $S^{\prime} \backslash S$.

(ii) $i=a\left(n_{3}-n_{2}\right), j=b n_{1}$ and $\delta_{0}(i)=a, \delta_{1}(j)=b$.

Proof. (i) $\Rightarrow$ (ii). Any element in $S^{\prime}$ can, by subtracting or adding multiples of $t_{0}, \ldots, t_{3}$, be changed into an element of the form $\left(a\left(n_{3}-n_{2}\right), b n_{1}\right)$ or $\left(a\left(n_{3}-\right.\right.$ $\left.\left.n_{1}\right), b n_{2}\right)$. For an element $\left(a\left(n_{3}-n_{1}\right), b n_{2}\right), \delta\left(a\left(n_{3}-n_{1}\right), b n_{2}\right)=a+\left(b n_{2}-\right.$ $\left.a n_{1}\right) / n_{3} \geq a$ if $b n_{2}-a n_{1} \geq 0$. For $b n_{2}-a n_{1}=c n_{3}, c<0$. from $b n_{2}+(-c) n_{3}$ $=a n_{1}$ and Remark $\left.2.1 \mathrm{~b}\right), a>b+(-c)$ or $a+c>b$. Thus in any case by Lemma $2.1\left(a\left(n_{3}-n_{1}\right), b n_{2}\right) \in S$. Now the $S$-minimality of $(i, j)$ implies (ii).

(ii) $\Rightarrow$ (i). $\quad \delta(i, j)=\delta\left(a\left(n_{3}-n_{2}\right), b n_{1}\right)=a+\left(b n_{1}-a n_{2}\right) / n_{3}, b n_{1}-a n_{2}=$ $c n_{3}$ and $c \geq 0$ would imply by Remark $2.1 \delta_{1}(j) \neq b$. Thus $c<0$ and $\delta(i, j)<$ $a=\delta_{0}(i)$, from which $(i, j) \notin S$ by Lemma 2.1. S-minimality now follows by observing that if $\left(i^{\prime}, j^{\prime}\right)=\left(a^{\prime}\left(n_{3}-n_{2}\right), b^{\prime} n_{1}\right), a^{\prime} \leq a, b^{\prime} \leq b$, then $\left(i^{\prime}, j^{\prime}\right) \mathbb{Z}_{S}$ $(i, j)$.

Remark 2.2. a) For another proof of Lemma 2.2 see Lemma 6 in [BSS]. From that Lemma 6 we also obtain that if $\left\{x_{1}^{\alpha_{1}}-x_{0}^{\alpha_{10}} x_{2}^{\alpha_{12}} x_{3}^{\alpha_{13}}, x_{2}^{\alpha_{0}}-x_{0}^{\alpha_{00}} x_{1}^{\alpha_{01}} x_{3}^{\alpha_{03}}\right\} \subseteq$ $\mathscr{B}\left(n_{1}, n_{2}, n_{3}\right)$, then $a<\alpha_{0}$ and $b<\alpha_{1}$, where $a$ and $b$ are as in Lemma 2.2 .

b) Note that if $(i, j)$ is $S^{\prime}$-minimal, then $(i, j)$ is also $S$-minimal.

Lemma 2.3. Let $(i, j)=\left(a\left(n_{3}-n_{2}\right)\right.$, bn $)$ be an $S$-minimal element of $S^{\prime} \backslash S$. Let $c=\left(b\left(n_{3}-n_{1}\right)-a\left(n_{3}-n_{2}\right)-n_{3}\right) / n_{3}$. Then $c$ is the largest nonnegative integer such that $(i, j)+c t_{0} \notin S$. Similarly, if $d=\left(a n_{2}-b n_{1}-n_{3}\right) / n_{3}$, then $d$ is the largest nonnegative integer such that $(i, j)+d t_{3} \notin S$. 
Proof. $\quad(i, j)+c t_{0}=\left(b\left(n_{3}-n_{1}\right)-n_{3}, b n_{1}\right)$, thus $\delta\left((i, j)+\left(c t_{0}\right)=b-1<\right.$ $b=\delta_{1}\left(b n_{1}\right)$ and therefore $(i, j)+c t_{0} \notin S$ by Lemma 2.1. As $(i, j)+(c+1) t_{0}$ $=b t_{1}, c$ is maximal. Proof of the second statement is obtained by symmetry.

Remark 2.3. In the sequel integers $c_{\imath}$ and $d_{\imath}$ will be defined for $\left(a_{\imath}\left(n_{3}-n_{2}\right)\right.$, $\left.b_{i} n_{1}\right)$ as were $c$ and $d$ for $\left(a\left(n_{3}-n_{2}\right), b n_{1}\right)$.

Lemma 2.4. Let $(i, j)=\left(a\left(n_{3}-n_{3}\right), b n_{1}\right)$ be an $S^{\prime}$-minimal element of $S^{\prime} \backslash S$ (see Remark $2.2 \mathrm{~b}$ ), and let $c$ and $d$ be as in Lemma 2.3. Then $(i, j)+c t_{0}+d t_{3} \notin$ S. Moreover, $\delta_{1}\left(b n_{1}+d n_{3}\right)=b+d$, and $\delta_{0}\left(a\left(n_{3}-n_{2}\right)+c n_{3}\right)=a+c$.

Proof. $\quad(i, j)+c t_{0}+d t_{3}=\left(b\left(n_{3}-n_{1}\right)-n_{3}, b n_{1}+d n_{3}\right)$, thus by Lemma 2.1 , it suffices to show $\delta_{1}\left(b n_{1}+d n_{3}\right)=b+d$, since the corresponding $\delta$-degree is $b-1+d$. Suppose $\delta_{1}\left(b n_{1}+d n_{3}\right)<b+d$, and let $b n_{1}+d n_{3}=\alpha_{1} n_{1}+\alpha_{2} n_{2}$ $+\alpha_{3} n_{3}$ with

$$
\alpha_{1}+\alpha_{2}+\alpha_{3}<b+d
$$

By Lemma $2.2 \delta_{1}\left(b n_{1}\right)=b$, hence $d>\alpha_{3}$, since otherwise $\left({ }^{*}\right)$ is contradicted, thus $b n_{1}+\left(d-\alpha_{3}\right)=\alpha_{1} n_{1}+\alpha_{2} n_{2} . \alpha_{1} \geq b$ implies $d-\alpha_{3}<\alpha_{1}-b+\alpha_{2}$ by Remark $2.1 \mathrm{~b}$ ), which contradicts $\left({ }^{*}\right)$. Thus $\alpha_{1}<b$ and $\alpha_{2}>0$. As $b n_{1}+d n_{3}=$ $a n_{2}-n_{3}$ by definition of $d, \alpha_{2}<a$. But then by a straightforward calculation $(i, j)+d t_{3}-\alpha_{2} t_{2}-\alpha_{3} t_{3}=\left(\left(a-\alpha_{2}\right)\left(n_{3}-n_{2}\right), \alpha_{1} n_{1}\right)<_{S^{\prime}}(i, j)$, contrary to $S^{\prime}$-minimality of $(i, j)$. The proof that $\delta_{0}\left(a\left(n_{3}-n_{2}\right)+c n_{3}\right)=a+c$ follows by symmetry.

Let $G^{\prime}=\left\{\left(a_{1}\left(n_{3}-n_{2}\right), b_{1} n_{1}\right) ; 1 \leq i \leq r\right\}$ be the $S^{\prime}$-minimal elements of $S^{\prime} \backslash S$, where $a_{1}<a_{2}<\cdots<a_{r}$ and $b_{1}>b_{2}>\cdots>b_{r}$. Let $a_{r+1}$ be the smallest positive integer satisfying $\delta_{0}\left(a_{r+1}\left(n_{3}-n_{2}\right)\right)<a_{r+1}$ and $b_{0}$ the smallest positive integer satisfying $\delta_{1}\left(b_{0} n_{1}\right)<b_{0}$. For each $i, 1 \leq i \leq r$, let $c_{i}=\left(b_{i}\left(n_{3}-n_{1}\right)-\right.$ $\left.a_{i}\left(n_{3}-n_{2}\right)-n_{3}\right) / n_{3}$ and $d_{i}=\left(a_{i} n_{2}-b_{i} n_{1}-n_{3}\right) / n_{3}$.

Remark 2.4. Let $\bar{R}=R / I\left(C\left(n_{1}, n_{2}, n_{3}\right)\right)$. By [BSS], $H_{m}^{1}(\bar{R}) \cong\left(\bar{R}_{\bar{x}_{0}} \cap \bar{R}_{\bar{x}_{3}}\right) / \bar{R}$ and the elements in $G^{\prime}$ correspond to the elements $\frac{\bar{x}_{2}^{a_{i}}}{\bar{x}_{3}^{d_{i}+1}} \equiv \frac{\bar{x}_{1}^{b_{i}}}{\bar{x}_{0}^{c_{i}+1}}$, obtained from the elements $x_{0}^{c_{i}+1} x_{2}^{a_{i}}-x_{1}^{b_{i}} x_{3}^{d_{i}+1}$ in $\mathscr{B}\left(n_{1}, n_{2}, n_{3}\right)$. Furthermore $a_{r+1}=\alpha_{0}$ and $b_{0}=$ $\alpha_{1}, \alpha_{0}$ and $\alpha_{1}$ defined in Remark 2.2 a). Also the $S$-minimal elements of $S^{\prime} \backslash S$ correspond to products of elements $\frac{\bar{x}_{2}^{a_{i}}}{\bar{x}_{3}^{d_{i}+1}}$. 
Lemma 2.5. For $1 \leq i \leq r, \delta_{1}\left(b_{i} n_{1}+\left(a_{i+1}-a_{i}-1\right) n_{2}\right)=b_{i}+a_{i+1}-a_{i}$ -1 , and $\delta_{0}\left(a_{i}\left(n_{3}-n_{2}\right)+\left(b_{i-1}-b_{i}-1\right)\left(n_{3}-n_{1}\right)\right)=a_{i}+b_{i-1}-b_{\imath}-1$.

Proof. Suppose we have

$$
b_{i} n_{1}+\left(a_{i+1}-a_{i}-1\right) n_{2}=\alpha_{1} n_{1}+\alpha_{2} n_{2}+\alpha_{3} n_{3},
$$

such that

$$
\alpha_{1}+\alpha_{2}+\alpha_{3}<b_{i}+\alpha_{i+1}-a_{i}-1
$$

By Lemma $2.2 a_{i+1}-a_{i}-1>\alpha_{2}$ (otherwise the minimality of $b_{i}$ is contradicted).

Case 1. $\alpha_{1} \geq b_{i}$. Starting with $\left({ }^{*}\right)$ a straightforward calculation gives $\left(a_{i+1}-\right.$ $\left.a_{i}-1-\alpha_{2}\right)\left(n_{3}-n_{2}\right)=\left(b_{i}+a_{i+1}-a_{i}-1-\alpha_{1}-\alpha_{2}-\alpha_{3}\right) n_{3}+\left(\alpha_{1}-b_{i}\right)\left(n_{3}-n_{1}\right)$. By the definition of $a_{r+1}, \delta_{0}\left(\left(a_{i+1}-a_{i}-1-\alpha_{2}\right)\left(n_{3}-n_{2}\right)\right)=a_{i+1}-a_{i}-1-$ $\alpha_{2}$, which contradicts Remark 2.1 b).

Case 2. $\alpha_{1}<b_{i}$. Then $\left(a_{i}\left(n_{3}-n_{2}\right), b_{i} n_{1}\right)+\left(a_{i+1}-a_{i}-1\right) t_{2}-\alpha_{2} t_{2}-\alpha_{3} t_{3}=$ $\left(\left(a_{i+1}-1-\alpha_{2}\right)\left(n_{3}-n_{2}\right), \alpha_{1} n_{1}\right)$ would be an $S$-minimal element of $S^{\prime} \backslash S$ by Lemma 2.2. But $a_{i}<a_{i+1}-1-\alpha_{2}<a_{i+1}$ and $\alpha_{1}<b_{i}$ thus such an element is neither $S^{\prime}$-minimal nor comparable to any $S^{\prime}$-minimal element by definition of $G^{\prime}$, a contradiction. Therefore $\delta_{1}\left(b_{i} n_{1}+\left(a_{i+1}-a_{i}-1\right) n_{2}\right)=b_{i}+a_{i+1}-a_{i}-1$.

Proof of the other statement follows by symmetry.

LEMMA 2.6. For $1 \leq i \leq r$, assume $0 \leq e<a_{i+1}-a_{i}, 0 \leq f<b_{i-1}-b_{i}$, and that there is no equation $\beta_{1} n_{1}+\beta_{2} n_{2}=\beta_{3} n_{3}$ with $\beta_{3}>0,0 \leq \beta_{1} \leq b_{1}+f, 0$ $\leq \beta_{2} \leq e$. Then $\delta_{1}\left(\left(b_{\imath}+f\right) n_{1}+e n_{2}\right)=b_{i}+f+e$ and $\delta_{0}\left(f\left(n_{3}-n_{1}\right)+\left(a_{i}+\right.\right.$ e) $\left.\left(n_{3}-n_{2}\right)\right)=a_{t}+e+f$.

Proof. Suppose $\left(b_{i}+f\right) n_{1}+e n_{2}=\alpha_{1} n_{1}+\alpha_{2} n_{2}+\alpha_{3} n_{3}$ with $\alpha_{1}+\alpha_{2}+\alpha_{3}$ $<b_{i}+e+f$. If $\alpha_{2} \geq e$, then $\delta_{1}\left(\left(b_{i}+f\right) n_{1}\right)<b_{i}+f<b_{i-1}$ would follow from the inequality, a contradiction to the definition of $b_{0}$. Thus $\alpha_{2}<e$. Similarly $\alpha_{1}<b_{i}+f$ since $0 \leq e<a_{i+1}-a_{i}<a_{i+1} \leq a_{r+1}$. Then $\left(b_{i}+f-\alpha_{1}\right) n_{1}+\left(e-\alpha_{2}\right) n_{2}=\alpha_{3} n_{3}$ contradicts the hypothesis. Hence $\delta_{1}\left(\left(b_{i}+f\right) n_{1}+e n_{2}\right)=b_{i}+f+e$.

Suppose $f\left(n_{3}-n_{1}\right)+\left(a_{i}+e\right)\left(n_{3}-n_{2}\right)=\alpha_{1}\left(n_{3}-n_{1}\right)+\alpha_{2}\left(n_{3}-n_{2}\right)+\alpha_{3} n_{3}$ with $\alpha_{1}+\alpha_{2}+\alpha_{3}<a_{i}+e+f$. As before, we would have $f>\alpha_{1}$. If $e \leq \alpha_{2}$, then $\delta_{0}\left(f\left(n_{3}-n_{1}\right)+a_{i}\left(n_{3}-n_{2}\right)\right)<f+a_{i}$ would contradict Lemma 2.5. Thus $e>\alpha_{2}$. Then $S^{\prime}$ contains $\left(a_{i}\left(n_{3}-n_{2}\right), b_{i} n_{1}\right)+f t_{1}+e t_{2}-\alpha_{1} t_{1}-\alpha_{2} t_{2}=\left(\alpha_{3} n_{3}\right.$, $\left.\left(b_{i}+f-\alpha_{1}\right) n_{1}+\left(e-\alpha_{2}\right) n_{2}\right)$, which contradicts the hypothesis. Therefore 
$\delta_{0}\left(f\left(n_{3}-n_{1}\right)+\left(a_{i}+e\right)\left(n_{3}-n_{2}\right)\right)=a_{i}+e+f$

LEMMA 2.7. For $1 \leq i \leq r$,

(i ) $\delta_{1}\left(b_{i} n_{1}+\left(a_{i+1}-a_{i}-1\right) n_{2}+d_{i} n_{3}\right)=b_{i}+a_{i+1}-a_{i}-1+d_{i}$, and

(ii) $\delta_{0}\left(a_{i}\left(n_{3}-n_{2}\right)+\left(b_{i-1}-b_{i}-1\right)\left(n_{3}-n_{1}\right)+c_{i} n_{3}\right)=a_{i}+b_{i-1}-b_{i}-1$ $+c_{i}$.

Proof. Suppose that $b_{i} n_{1}+\left(a_{i+1}-a_{i}-1\right) n_{2}+d_{i} n_{3}=\alpha_{1} n_{1}+\alpha_{2} n_{2}+\alpha_{3} n_{3}$ with $\alpha_{1}+\alpha_{2}+\alpha_{3}<b_{i}+a_{i+1}-a_{i}-1+d_{i}$. By Lemma $2.5 d_{i}>\alpha_{3}$. Suppose $\alpha_{2} \leq a_{i+1}-a_{i}-1$. Then $\left(a_{i+1}-a_{i}-1-\alpha_{2}\right) n_{2}+\left(d_{i}-\alpha_{3}\right) n_{3}=\left(\alpha_{1}-b_{i}\right) n_{1}$ and $\alpha_{1}-b_{i}<\left(a_{i+1}-a_{i}-1-\alpha_{2}\right)+d_{i}-\alpha_{3}$ by the initially assumed inequality, a contradiction to Remark 2.1b). Thus $a_{i+1}-a_{i}-1<\alpha_{2}$ and $b_{i} n_{1}+d_{i} n_{3}=$ $\alpha_{1} n_{1}+\left(\alpha_{2}-a_{i+1}+a_{i}+1\right) n_{2}+\alpha_{3} n_{3}$, which is contrary to the second part of Lemma 2.4. Therefore (i) holds and the proof of (ii) follows by symmetry.

LEMMA 2.8. For $1 \leq i \leq r, \delta_{0}\left(\left(a_{i+1}-1\right)\left(n_{3}-n_{2}\right)+c_{i} n_{3}\right)=a_{i+1}-1+c_{i}$ and $\delta_{1}\left(\left(b_{i-1}-1\right) n_{1}+d_{i} n_{3}\right)=b_{i-1}-1+d_{i}$. Moreover each of $\left(a_{i}\left(n_{3}-n_{2}\right), b_{i} n_{1}\right)$ $+\left(a_{i+1}-a_{i}-1\right) t_{2}+c_{i} t_{0}+d_{i} t_{3},\left(a_{i}\left(n_{3}-n_{2}\right), b_{i} n_{1}\right)+\left(b_{i-1}-b_{i}-1\right) t_{1}+c_{i} t_{0}$ $+d_{i} t_{3}$ is in $S^{\prime} \backslash S$.

Proof. By substituting for $d_{i} n_{3}$ we obtain $\delta\left(\left(a_{i}\left(n_{3}-n_{2}\right), b_{i} n_{1}\right)+\left(b_{i-1}-b_{i}\right.\right.$ $\left.-1) t_{1}+c_{i} t_{0}+d_{i} t_{3}\right)=\left(a_{i}\left(n_{3}-n_{2}\right)+b_{i} n_{1}+\left(b_{i-1}-b_{i}-1+c_{i}\right) n_{3}+a_{i} n_{2}-\right.$ $\left.b_{i} n_{1}-n_{3}\right) / n_{3}=a_{i}+b_{i-1}-b_{i}-1+c_{i}-1$. It follows by Lemma 2.7 (ii) and Lemma 2.1 that this element is not in $S$. But then, substituting for $c_{i} n_{3}, \delta\left(\left(a_{i}\left(n_{3}\right.\right.\right.$ $\left.\left.\left.-n_{2}\right), b_{i} n_{1}\right)+\left(b_{i-1}-b_{i}-1\right) t_{1}+c_{i} t_{0}+d_{i} t_{3}\right)=\left(a_{i}\left(n_{3}-n_{2}\right)+b_{i} n_{1}+\left(b_{i-1}-\right.\right.$ $\left.\left.b_{i}-1+d_{i}\right) n_{3}+b_{i}\left(n_{3}-n_{1}\right)-d_{i}\left(n_{3}-n_{2}\right)-n_{3}\right) / n_{3}=b_{i-1}-2+d_{i}$. Thus $\delta_{1}\left(\left(b_{i-1}\right.\right.$ $\left.-1) n_{1}+d_{i} n_{3}\right)>b_{i-1}-2+d_{i}$ by Lemma 2.1. Since $\delta_{1}\left(\left(b_{i-1}-1\right) n_{1}+d_{i} n_{3}\right)$ $\leq b_{i-1}-1+d_{i}$, equality holds.

Proof of the other two statements follows by symmetry.

Proposition 2.1. Let $1 \leq i \leq r$. Assume $0 \leq e<a_{i+1}-a_{i}, 0 \leq f<b_{i-1}-$ $b_{i}$ and that there is no equation $\beta_{1} n_{1}+\beta_{2} n_{2}=\beta_{3} n_{3}$ with $\beta_{3}>0,0 \leq \beta_{1} \leq b_{i}+f$, $0 \leq \beta_{2} \leq e$. Then $\left(a_{i}\left(n_{3}-n_{2}\right), b_{i} n_{1}\right)+f t_{1}+e t_{2}+c_{i} t_{0}+d_{i} t_{3}$ is in $S^{\prime} \backslash S$.

Proof. Suppose $\left(b_{i}+f\right) n_{1}+e n_{2}+d_{i} n_{3}=\alpha_{1} n_{1}+\alpha_{2} n_{2}+\alpha_{3} n_{3}$ and $\alpha_{1}+\alpha_{2}$ $+\alpha_{3}<b_{i}+f+e+d_{i}$. Lemma 2.6 implies $d_{i}>\alpha_{3}$ and thus $e<\alpha_{2}$ by Remark 2.1 b). But then $\left(b_{i}+f\right) n_{1}+d_{i} n_{3}=\alpha_{1} n_{1}+\left(\alpha_{2}-e\right) n_{2}+\alpha_{3} n_{3}$, which contradicts Lemma 2.8. Therefore $\delta_{1}\left(\left(b_{i}+f\right) n_{1}+e n_{2}+d_{i} n_{3}\right)=b_{i}+f+e+d_{i}$. Then, 
substituting for $c_{i} n_{3}$, one obtains $\delta\left(\left(a_{i}\left(n_{3}-n_{2}\right), b_{1} n_{1}\right)+f t_{1}+e t_{2}+c_{i} t_{0}\right.$ $\left.+d_{i} t_{3}\right)=\left(a_{i}\left(n_{3}-n_{2}\right)+b_{i} n_{1}+\left(f+e+d_{i}\right) n_{3}+b_{i}\left(n_{3}-n_{2}\right)-a_{i}\left(n_{3}-n_{2}\right)-\right.$ $\left.n_{3}\right) / n_{3}=b_{i}+f+e+d_{i}-1$, from which the conclusion follows by Lemma 2.1.

Lemma 2.9. Assume that $\left(a\left(n_{3}-n_{2}\right), b n_{1}\right)$ is an $S$-minimal element of $S^{\prime} \backslash S$ which is not $S^{\prime}$-minimal. Let $c=\left(b\left(n_{3}-n_{2}\right)-a n_{1}-n_{3}\right) / n_{3}$ and $d=\left(a n_{2}-b n_{1}\right.$ $\left.-n_{3}\right) / n_{3}$. Then either $\delta_{1}\left(b n_{1}+d n_{3}\right)<b+d$ or $\delta_{0}\left(a\left(n_{3}-n_{2}\right)+c n_{3}\right)<a+c$.

Proof. By symmetry we can assume without loss of generality $a \leq b$. (This will imply the first of the two stated inequalities, the other follows from $a>b$.) By $S$-minimality assume $\left(a\left(n_{3}-n_{2}\right), b n_{1}\right)=\sum_{j=1}^{t}\left(a_{i(j)}\left(n_{3}-n_{2}\right), b_{i(j)} n_{1}\right)$, where each summand is an $S^{\prime}$-minimal element of $S^{\prime} \backslash S$ and $t \geq 2 . \sum_{j=1}^{t} a_{\imath^{(j)}}=a \leq b=$ $\sum_{j=1}^{t} b_{\imath(j)}$ implies that there exists an $h, 1 \leq h \leq t$, such that $a_{i(h)} \leq b_{\imath(h)}$ (other. wise $a>b$ ). W.lo.g. let $h=1$. Then $b n_{1}+d n_{3}=b n_{1}+a n_{2}-b n_{1}-n_{3}=$ $a n_{2}-n_{3}=\sum_{j=1}^{t} a_{i(j)} n_{2}-n_{3}=\sum_{j=2}^{t} b_{i(j)} n_{1}+\left(\sum_{j=1}^{t} a_{i(j)} n_{2}-\sum_{j=2}^{t} b_{i(j)} n_{1}\right)-n_{3}=$ $a_{i(1)} n_{2}+\sum_{j=2}^{t} b_{i(j)} n_{1}+\sum_{j=2}^{t}\left(a_{\imath(j)} n_{2}-b_{\imath(j)} n_{1}-n_{3}\right)+(t-1) n_{3}-n_{3}=a_{\imath(1)} n_{2}+$ $\sum_{j=2}^{t} b_{i(j)} n_{1}+\left(t-2+\sum_{j=2}^{t} d_{i(j)}\right) n_{3}$. The definition of $b_{0}$ and $b_{i(j)}, 1 \leq j \leq t$, implies $d>(t-2)+\sum_{j=2}^{t} d_{i(j)}$. From this and $b_{i(1)} \geq a_{t(1)}$ (thus $b \geq a_{i(1)}+$ $\left.\sum_{j=2}^{t} b_{i(j)}\right), \delta_{1}\left(b n_{1}+d n_{3}\right)<b+d$ is obtained.

Lemma 2.10. Let $(A, B)$ be an $S$-maximal element of $S^{\prime} \backslash S$, where $A=A_{1}\left(n_{3}\right.$ $\left.-n_{1}\right)+A_{2}\left(n_{3}-n_{2}\right)+A_{3} n_{3}, B=B_{1} n_{1}+B_{2} n_{2}+B_{3} n_{3} \quad$ with $\delta_{0}(A)=\sum_{i=1}^{3} A_{i}$, $\delta_{1}(B)=\sum_{i=1}^{3} B_{i}$. Then for some $i, 1 \leq i \leq r, A_{2}-B_{2}=a_{\imath}, B_{1}-A_{1}=b_{\imath}, A_{3}=$ $c_{\imath}$, and $B_{3}=d_{\imath}$.

Proof. By Lemma $2.1 \delta_{1}(B)>\delta(A, B)$. If $\delta_{1}(B)>\delta(A, B)+1$, then $\delta_{1}(B)>\delta\left((A, B)+t_{0}\right)$, and then $(A, B)<_{S}(A, B)+t_{0} \in S^{\prime}$, contrary to $S$-maximality. Thus $B_{1}+B_{2}+B_{3}=\delta_{1}(B)=\delta(A, B)+1$. Then $\left(B_{1}+B_{2}+\right.$ $\left.B_{3}-1\right) n_{3}=\delta(A, B) n_{3}=A_{1}\left(n_{3}-n_{1}\right)+A_{2}\left(n_{3}-n_{2}\right)+A_{3} n_{3}+B_{1} n_{1}+B_{2} n_{2}+$ $B_{3} n_{3}$, which implies $A_{1}\left(n_{3}-n_{1}\right)+A_{2}\left(n_{3}-n_{2}\right)+A_{3} n_{3}=B_{1}\left(n_{3}-n_{1}\right)+B_{2}\left(n_{3}-\right.$ $\left.n_{2}\right)-n_{3}$. Thus $A_{3}=\left(\left(B_{1}-A_{1}\right)\left(n_{3}-n_{1}\right)-\left(A_{2}-B_{2}\right)\left(n_{3}-n_{2}\right)-n_{3}\right) / n_{3}$. By symmetry, $B_{3}=\left(\left(A_{2}-B_{2}\right) n_{2}-\left(B_{1}-A_{1}\right) n_{1}-n_{3}\right) / n_{3}$. Next consider the following element of $S^{\prime} \backslash S:(A, B)-A_{3} t_{0}-B_{3} t_{3}=\left(A_{1}\left(n_{3}-n_{1}\right)+A_{2}\left(n_{3}-n_{2}\right), B_{1} n_{1}\right.$ $\left.+B_{2} n_{2}\right)$. Suppose $A_{1} \geq B_{1}$. Then $\left(\left(A_{1}-B_{1}\right)\left(n_{3}-n_{1}\right)+A_{2}\left(n_{3}-n_{2}\right), B_{2} n_{2}\right) \leq_{S}$ $(A, B) . A_{2} \geq B_{2}$ immediately implies $(A, B) \in S$, contrary to hypothesis. If $A_{2}$ $<B_{2}$ then $\left(\left(A_{1}-B_{1}\right)\left(n_{3}-n_{1}\right),\left(B_{2}-A_{2}\right) n_{2}\right) \in S$ by Remark 2.1 b) and Lemma 2.1 since $\delta_{0}(A)=A_{1}+A_{2}+A_{3}$. But then again $(A, B) \in S$ contrary to assumption. Thus $A_{1}<B_{1}$. By symmetry $B_{2}<A_{2}$. Hence, by Remark 2.1 a), we must 
have $\left(\left(A_{2}-B_{2}\right)\left(n_{3}-n_{2}\right),\left(B_{1}-A_{1}\right) n_{1}\right) \in S^{\prime}$ with $\delta\left(\left(A_{2}-B_{2}\right)\left(n_{3}-n_{2}\right)\right)=$ $A_{2}-B_{2}$ and $\delta_{1}\left(\left(B_{1}-A_{1}\right) n_{1}\right)=B_{1}-A_{1}$. By Lemma $2.2\left(\left(A_{2}-B_{2}\right)\left(n_{3}-n_{2}\right)\right.$, $\left.\left(B_{1}-A_{1}\right) n_{1}\right)$ is an $S$-minimal element of $S^{\prime} \backslash S$. But $\delta_{0}\left(\left(A_{2}-B_{2}\right)\left(n_{3}-n_{2}\right)+\right.$ $\left.A_{3} n_{3}\right)=A_{2}-B_{2}+A_{3}$ and $\delta_{1}\left(\left(B_{1}-A_{1}\right) n_{1}+B_{3} n_{3}\right)=B_{1}-A_{1}+B_{3}$, thus by Lemma 2.9, $\left(\left(A_{2}-B_{2}\right)\left(n_{3}-n_{2}\right),\left(B_{1}-A_{1}\right) n_{1}\right)$ is an $S^{\prime}$-minimal element of $S^{\prime} \backslash S$. Therefore for some $i, 1 \leq i \leq r, A_{2}-B_{2}=a_{i}$, and $B_{1}-A_{1}=b_{i} . A_{3}=c_{i}$ and $B_{3}=d_{i}$ now follow from the definition of $c_{i}$ and $d_{i}$ and the calculations for $A_{3}$ and $B_{3}$ above.

Proposition 2.2. Let $(A, B)$ be an $S$-maximal element of $S^{\prime} \backslash S$. Then $(A, B)$ $=\left(a_{i}\left(n_{3}-n_{2}\right), b_{i} n_{1}\right)+f t_{1}+e t_{2}+c_{i} t_{0}+d_{i} t_{3}$ where:

(i) $\left(a_{i}\left(n_{3}-n_{2}\right), b_{i} n_{1}\right)$ is an $S^{\prime}$-minimal element of $S^{\prime} \backslash S$.

(ii) $c_{i}$ and $d_{i}$ are as defined before, namely $c_{i}=\left(b_{i}\left(n_{3}-n_{1}\right)-a_{i}\left(n_{3}-n_{2}\right)-n_{3}\right) / n_{3}$, $d_{i}=\left(a_{i} n_{2}-b_{i} n_{1}-n_{3}\right) / n_{3}$.

(iii) $0 \leq e<a_{i+1}-a_{i}, 0 \leq f<b_{i-1}-b_{i}$ and there is no equation $\beta_{1} n_{1}+\beta_{2} n_{2}=$ $\beta_{3} n_{3}, 0 \leq \beta_{1} \leq b_{i}+f, 0 \leq \beta_{2} \leq e$.

Thus every $S$-maximal element is one of the elements in Proposition 2.1.

Proof. We use the notation of Lemma 2.10. Thus for some $i, 1 \leq i \leq r,(A, B)$ $=\left(a_{i}\left(n_{3}-n_{2}\right), b_{i} n_{1}\right)+A_{1} t_{1}+B_{2} t_{2}+c_{i} t_{0}+d_{i} t_{3}$. It suffices to show that $A_{1}$ and $B_{2}$ satisfy the conditions for $f$ and $e$ in (iii) above. Suppose $A_{1} \geq b_{i-1}-b_{i}$. Consider $\left(a_{i}\left(n_{3}-n_{2}\right), b_{i} n_{1}\right)+\left(b_{i-1}-b_{i}\right)\left(n_{3}-n_{1}, n_{1}\right)=\left(a_{i}\left(n_{3}-n_{2}\right)+\left(b_{i-1}-b_{i}\right)\right.$ $\left.\left(n_{3}-n_{1}\right), b_{i-1} n_{1}\right) \in S^{\prime} \backslash S$.

Case 1. $i=1$. Then $\delta_{1}\left(b_{i-1} n_{1}\right)=\delta_{1}\left(b_{0} n_{1}\right)<b_{0}$ and $\delta_{1}\left(B_{1} n_{1}+B_{2} n_{2}+\right.$ $\left.B_{3} n_{3}\right)<B_{1}+B_{2}+B_{3}$ since $B_{1}=B_{1}-A_{1}+A_{1}=b_{1}+A_{1} \geq b_{0}$, contrary to assumption.

Case 2. $2 \leq i \leq r$. Since $\left(a_{i-1}\left(n_{3}-n_{2}\right), b_{i-1} n_{1}\right) \in S^{\prime} \backslash S$ and $a_{i-1}<a_{i}$, $\left(a_{i-1}\left(n_{3}-n_{2}\right), b_{i-1} n_{1}\right)<_{S}\left(a_{i}\left(n_{3}-n_{2}\right)+\left(b_{i-1}-b_{i}\right)\left(n_{3}-n_{1}\right), b_{i-1} n_{1}\right) . \quad$ Thus $a_{i}\left(n_{3}-n_{2}\right)+\left(b_{i-1}-b_{i}\right)\left(n_{3}-n_{1}\right)=a_{i-1}\left(n_{3}-n_{2}\right)+g n_{3}, g>0$. As $a_{i-1}<a_{i}$, $\delta_{0}\left(a_{i}\left(n_{3}-n_{2}\right)+\left(b_{i-1}-b_{i}\right)\left(n_{3}-n_{3}\right)\right)<a_{i}+b_{i-1}-b_{i}$ and $\delta_{0}\left(A_{1}\left(n_{3}-n_{1}\right)+\right.$ $\left.A_{2}\left(n_{3}-n_{2}\right)+A_{3} n_{3}\right)<A_{1}+A_{2}+A_{3}$ since $A_{2} \geq a_{i}$, which is contrary to assumption. Therefore $A_{1}<b_{i-1}-b_{i}$ and by symmetry $B_{2}<a_{i+1}-a_{i}$. Finally if $\beta_{1} n_{1}+$ $\beta_{2} n_{2}=\beta_{3} n_{3}$ with $\beta_{3}>0,0 \leq \beta_{1} \leq b_{1}+A_{1}=B_{1}, 0 \leq \beta_{2} \leq B_{2}$, then $\delta_{1}\left(B_{1} n_{1}+\right.$ $\left.B_{2} n_{2}+B_{3} n_{3}\right)<B_{1}+B_{2}+B_{3}$, a contradiction to the choice of $B_{i}, 1 \leq i \leq 3$. Hence there is no such equation.

Proposition 2.3. Let $(A, B)$ be an $S$-maximal element in $S^{\prime} \backslash S$ and $\left(A_{0}\right.$, $\left.B_{0}\right)$ an $S^{\prime}$-minimal element of $S^{\prime} \backslash S$. Then $\left(A_{0}, B_{0}\right) \leq_{S}(A, B)$. 
Proof. By Proposition 2.2 let $(A, B)=\left(a_{i}\left(n_{3}-n_{2}\right), b_{i} n_{1}\right)+f t_{1}+e t_{2}+c_{i} t_{0}$ $+d_{i} t_{3}$ and let $\left(A_{0}, B_{0}\right)=\left(a_{j}\left(n_{3}-n_{2}\right), b_{j} n_{1}\right)$. If $i=j$ there is nothing to prove. Assume $i \neq j$. By symmetry we can assume $b_{j}<b_{i}$ and $a_{j}>a_{i}$ (for $b_{j}>b_{i}$ we use $\left.a_{j}<a_{i}\right)$. Then $\left(A_{0}, B_{0}\right)+\left(b_{i}-b_{j}\right)\left(n_{3}-n_{1}, n_{1}\right)=\left(a_{j}\left(n_{3}-n_{2}\right)+\left(b_{i}-\right.\right.$ $\left.\left.b_{j}\right)\left(n_{3}-n_{1}\right), b_{i} n_{1}\right) \in S^{\prime} .\left(a_{i}\left(n_{3}-n_{2}\right), b_{i} n_{1}\right) \in S^{\prime}$ implies $\left(a_{j}-a_{i}\right)\left(n_{3}-n_{2}\right)+$ $\left(b_{i}-b_{j}\right)\left(n_{3}-n_{1}\right)=g n_{3}, g>0$, thus $a_{j}\left(n_{3}-n_{2}\right)+\left(b_{i}-b_{j}\right)\left(n_{3}-n_{1}\right)=a_{i}\left(n_{3}\right.$ $\left.-n_{2}\right)+g n_{3}$. Thus $\left(a_{j}\left(n_{3}-n_{2}\right)+\left(b_{i}-b_{j}\right)\left(n_{3}-n_{1}\right), b_{i} n_{1}\right)=\left(a_{i}\left(n_{3}-n_{2}\right)+\right.$ $\left.g n_{3}, b_{i} n_{1}\right)$ for some $g>0$. If $\left(a_{i}\left(n_{3}-n_{2}\right)+g n_{3}, b_{i} n_{1}\right) \in S$, then $g>c_{i}$ by Lemma 2.3. Then $a_{j}\left(n_{3}-n_{2}\right)+\left(b_{i}-b_{j}\right)\left(n_{3}-n_{1}\right) \geq a_{i}\left(n_{3}-n_{2}\right)+\left(c_{i}+1\right) n_{3}$ $=b_{i}\left(n_{3}-n_{1}\right)$ (by definition of $\left.c_{i}\right)$. From this $a_{j}\left(n_{3}-n_{2}\right) \geq b_{j}\left(n_{3}-n_{1}\right)$, from which $\left(a_{j}\left(n_{3}-n_{2}\right), b_{j} n_{1}\right) \in S$ by Lemma 2.1 since then $a_{j}+\left(-a_{j} n_{2}+b_{j} n_{1}\right) / n_{3}$ $\geq b_{j}$. But this is contrary to assumption. Hence $\left(a_{i}\left(n_{3}-n_{2}\right)+g n_{3}, b_{i} n_{1}\right) \notin S$ and $g \leq c_{i}$. Therefore $\left(A_{0}, B_{0}\right) \leq_{S}\left(A_{0}, B_{0}\right)+\left(b_{i}-b_{j}\right)\left(n_{3}-n_{1}, n_{1}\right) \leq_{S}\left(a_{i}\left(n_{3}-n_{2}\right)\right.$, $\left.b_{i} n_{1}\right)+c_{i} t_{0} \leq_{S}(A, B)$, which finishes the proof.

We can now state and prove the following.

THEOREM 2.1. For a monomial curve $C\left(n_{1}, n_{2}, n_{3}\right)$ in $P_{K}^{3}$ we have $k\left(C\left(n_{1}, n_{2}\right.\right.$, $\left.\left.n_{3}\right)\right)=\operatorname{diam}\left(H_{\underline{m}}^{1}(\bar{R})\right)$. Furthermore an element of minimal degree in $H_{\underline{m}}^{1}(\bar{R})$ must occur amongst the $S^{\prime}$-minimal elements, thus by Remark 2.4, amongst the generators of $H_{\underline{m}}^{1}(\bar{R})$ obtained from elements $x_{0}^{c_{i}+1} x_{2}^{a_{i}}-x_{1}^{b_{i}} x_{3}^{d_{i}+1} \in \mathscr{B}\left(n_{1}, n_{2}, n_{3}\right)$. An element of maximal degree is obtained by considering all possible extensions of $S^{\prime}$-minimal elements to $S$-maximal elements.

Proof. The first statement follows immediately from Proposition 2.3, since $(A, B)$ and $\left(A_{0}, B_{0}\right)$ are arbitrary in their respective sets. The second statement is a consequence of the correspondence between $S^{\prime}$-minimal elements of $S^{\prime} \backslash S$ and generators of $H_{\underline{m}}^{1}(\bar{R})$ as specified by Remark 2.4. The third statement follows immediately from Proposition 2.3.

\section{An algorithm for computing $k\left(C\left(n_{1}, n_{2}, n_{3}\right)\right)$}

This section requires a somewhat more detailed study of the minimal generating set $\mathscr{B}=\mathscr{B}\left(n_{1}, n_{2}, n_{3}\right)$ of $I(C)$ obtained in [BR] (see also [B2] and [BH]). For the convenience of the reader not familiar with the algorithm of $[B R]$, we also include a brief description of it at the end of this section when we compute $k\left(C\left(n_{1}, n_{2}\right.\right.$, $\left.n_{3}\right)$ ). Let

$$
\mathscr{B}_{2}=\left\{\beta_{2} ; \beta_{2}=x_{0}^{a_{0}} x_{2}^{a_{2}}-x_{1}^{a_{1}} x_{3}^{a_{3}} \in \mathscr{B}\left(n_{1}, n_{2}, n_{3}\right) \text {, and } a_{i}>0,0 \leq i \leq 3\right\} .
$$


From elements in $\mathscr{B}_{2}, \frac{x_{2}^{a_{2}}}{x_{3}^{a_{3}}} \equiv \frac{x_{1}^{a_{1}}}{x_{0}^{a_{0}}} \in H_{\underline{m}}^{1}(\bar{R})$ are obtained and these elements correspond to the $S^{\prime}$-minimal elements of $S^{\prime} \backslash S, S$ and $S^{\prime}$ defined in Section 2. (By abuse of notation we shall delete bars on variables.) Let

$$
\mathscr{B}_{3}=\left\{\beta_{3} ; \beta_{3}=x_{0}^{b_{0}} x_{3}^{b_{3}}-x_{1}^{b_{1}} x_{2}^{b_{2}} \in \mathscr{B}\left(n_{1}, n_{2}, n_{3}\right) \text {, and } b_{i}>0,0 \leq i \leq 3\right\} \text {. }
$$

Then $\mathscr{B}\left(n_{1}, n_{2}, n_{3}\right)=\mathscr{B}_{2} \cup \mathscr{B}_{3} \cup\left\{F_{1}=x_{1}^{\alpha_{1}}-x_{0}^{\alpha_{10}} x_{2}^{\alpha_{12}} x_{3}^{\alpha_{13}}, F_{2}=x_{2}^{a_{0}}-x_{0}^{\alpha_{00}} x_{1}^{\alpha_{01}} x_{3}^{\alpha_{03}}\right\}$ and every binomial $\beta \in \mathscr{B}\left(n_{1}, n_{2}, n_{3}\right)$, except for the first two binomials in the algorithm of $[\mathrm{BR}]$, is obtained from binomials $\beta^{\prime}$ and $\beta^{\prime \prime}$ by cross-multiplying the monomial terms of $\beta^{\prime}$ with the monomial terms of $\beta^{\prime \prime}$ and deleting common factors of the resulting two monomials. We will write for this $\beta^{\prime \prime}\left(\beta^{\prime}\right)=\beta$ and say $\beta^{\prime \prime}$ acts on $\beta^{\prime}$ to produce $\beta$.

Definition 3.1. Let $\{i, j\}=\{2,3\}, \beta_{j} \in \mathscr{B}_{j}$. The set

$$
B\left(\beta_{j}\right)=\left\{\beta_{i} ; \beta_{j}\left(\bar{\beta}_{i}\right)=\beta_{i},\left\{\beta_{i}, \bar{\beta}_{i}\right\} \subseteq \mathscr{B}_{i}\right\} \cup\left\{\bar{\beta}_{i} ; \beta_{j}\left(\bar{\beta}_{i}\right)=\beta_{i},\left\{\beta_{i}, \bar{\beta}_{i}\right\} \subseteq \mathscr{B}_{i}\right\},
$$

will be called a block in $\mathscr{B}_{i}$.

Ordering the elements in $\mathscr{B}_{i}, i=1,2$ by decreasing $x_{0}$ and $x_{1}$ exponents (and therefore increasing $x_{2}$ and $x_{3}$ exponents) establishes by [BR] a linear order on each block and on $\mathscr{B}_{i}$.

Definition 3.2. $\beta_{i} \in B\left(\beta_{j}\right)$ of largest or smallest $x_{0}$ and $x_{1}$ exponent are called end polynomials. All other $\beta_{i} \in B\left(\beta_{j}\right)$ are called middle polynomials.

Definition 3.3. Let $x_{0}^{a_{0}} x_{2}^{a_{2}}-x_{1}^{a_{1}} x_{3}^{a_{3}} \in \mathscr{B}_{2}, X$ a monomial term in $R, 0 \neq$ $X \frac{x_{2}^{a_{2}}}{x_{3}^{a_{3}}} \in H_{\underline{m}}^{1}(\bar{R})$. Then $d\left(\frac{x_{2}^{a_{2}}}{x_{3}^{a_{3}}}\right)=d(X)+a_{2}+a_{3}$, where $d(X)$ is the ordinary total degree of a monomial term.

Remark 3.1. Note that $d\left(\frac{x_{2}^{a_{2}}}{x_{3}^{a_{3}}}\right)=\delta\left(a_{2}\left(n_{3}-n_{2}\right), a_{1} n_{1}\right)$ and if $t_{i}$ and $x_{i}$ correspond, $0 \leq i \leq 3$, then $d$ and $\delta$ agree.

Definition 3.4. For $x_{0}^{a_{0}} x_{2}^{a_{2}}-x_{1}^{a_{1}} x_{3}^{a_{3}} \in \mathscr{B}_{2}$ and $X$ a monomial term in $R, 0 \neq$ $X \frac{x_{2}^{a_{2}}}{x_{3}^{a_{3}}} \in H_{\underline{m}}^{1}(\bar{R})$ is a maximal multiple of $\frac{x_{2}^{a_{2}}}{x_{3}^{a_{3}}}$ if $x_{i}\left(X \frac{x_{2}^{a_{2}}}{x_{3}^{a_{3}}}\right)=0$ in $H_{\underline{m}}^{1}(\bar{R}), 0 \leq$ $i \leq 3$. 
Remark 3.2. Note that if $\beta_{2}=x_{0}^{a_{0}} x_{2}^{a_{2}}-x_{1}^{a_{1}} x_{3}^{a_{3}}$ is a middle polynomial in $\mathscr{B}_{2}$, then there exists a uniquely determined polynomial $x_{0}^{b_{0}} x_{3}^{b_{3}}-x_{1}^{b_{1}} x_{2}^{b_{2}} \in \mathscr{B}_{3}$, which acts on $\beta_{2}$ to produce the successor of $\beta_{2}$ in $\mathscr{B}_{2}$. If $\beta_{2}$ is an end polymomial in $\mathscr{B}_{2}$, but neither the first nor the last polynomial in $\mathscr{B}_{2}$, then $B\left(\beta_{2}\right) \neq \emptyset$ and if $B\left(\beta_{2}\right)=\left\{f_{1}=x_{0}^{b_{0}} x_{3}^{b_{3}}-x_{1}^{b_{1}} x_{2}^{b_{2}}, \cdots, f_{1}=x_{0}^{c_{0}} x_{3}^{c_{3}}-x_{1}^{c_{1}} x_{2}^{c_{2}}\right\}$ with $f_{1}$ the first and $f_{l}$ the last polynomial in $B\left(\beta_{2}\right)$, then $f_{1}$ acts on the predecessor of $\beta_{2}$ to produce $\beta_{2}$ and $f_{1}$ acts on $\beta_{2}$ to produce the successor of $\beta_{2}$ in $\mathscr{B}_{2}$. If $\beta_{2}$ is the first or last polynomial in $\mathscr{B}_{2}$, then $B\left(\beta_{2}\right)$ may be empty. If for this case $B\left(\beta_{2}\right)$ is empty, then let $x_{0}^{b_{0}} x_{3}^{b_{3}}-x_{1}^{b_{1}} x_{2}^{b_{2}}=x_{0}^{c_{0}} x_{3}^{c_{3}}-x_{1}^{c_{1}} x_{2}^{c_{2}}$ be the first or the last polynomial in $\mathscr{B}_{3}$, respectively.

Now let $m=x_{0}^{\gamma_{0}} x_{3}^{\gamma_{3}} \frac{x_{2}^{a_{2}}}{x_{3}^{a_{3}}} x_{1}^{\gamma_{1}} x_{2}^{\gamma_{2}}$ be an arbitrary maximal multiple. Assume $\frac{x_{2}^{a_{2}}}{x_{3}^{a_{3}}}$ corresponds to a middle polynomial. If $\gamma_{1} \geq b_{1}, b_{1}$ defined in Remark 3.2 , then, since by the algorithm of $[\mathrm{BR}] a_{2}>b_{2}$,

$$
m=x_{0}^{\gamma_{0}} x_{3}^{\gamma_{3}} \frac{x_{2}^{a_{2}}}{x_{3}^{a_{3}}} x_{1}^{\gamma_{1}} x_{2}^{\gamma_{2}}=x_{0}^{\gamma_{0}+b_{0}} x_{3}^{\gamma_{3}} \frac{x_{2}^{a_{2}-b_{2}}}{x_{3}^{a_{3}-b_{3}}} x_{1}^{\gamma_{1}-b_{1}} x_{2}^{\gamma_{2}}
$$

where $\frac{x_{2}^{a_{2}-b_{2}}}{x_{3}^{a_{3}-b_{3}}}$ corresponds to the predecessor of $\frac{x_{2}^{a_{2}}}{x_{3}^{a_{3}}}$. Note that the $x_{2}$-exponent is unchanged. If $\gamma_{2} \geq b_{2}$, then by the exactly analogous procedure, with $\frac{x_{2}^{a_{2}}}{x_{3}^{a_{3}}}$ represented by $\frac{x_{1}^{a_{1}}}{x_{0}^{a_{0}}}$, we obtain a reduction of $\gamma_{2}$ by $b_{2}$ and a shift to the successor of $\frac{x_{2}^{a_{2}}}{x_{3}^{a_{3}}}$. Now let $\frac{x_{2}^{a_{2}}}{x_{3}^{a_{3}}}$ correspond to an end polynomial. If $\gamma_{1} \geq b_{1}$, again $b_{1}$ defined in Remark 3.2, then as above $a_{2}>b_{2}$, and we have a reduction in $m$ of the $x_{1}$-exponent by $b_{1}$ and a shift to the predecessor. Note that if $\frac{x_{2}^{a_{2}}}{x_{3}^{a_{3}}}$ cprresponds to the first element in $\mathscr{B}_{2}$, then $\gamma_{1} \geq b_{1}$ is not possible, since this would imply $m=0$. If $\gamma_{2} \geq c_{2}, c_{2}$ defined in Remark 3.2 then as before we obtain a reduction of $\gamma_{2}$ in $m$ by $c_{2}$ and a shift to the successor. Note again that if $\frac{x_{2}^{a_{2}}}{x_{3}^{a_{3}}}$ corresponds to the last polynomial in $\mathscr{B}_{2}$, then this is not possible.

Since this procedure leaves one of the two exponents $\gamma_{i}, i=1,2$, invariant, while reducing the other we get a reduction of maximal multiples to maximal multiples to one of two possible cases: 
I. $m^{*}=x_{0}^{\sigma_{0}} x_{3}^{\sigma_{3}} \frac{x_{2}^{a_{2}}}{x_{3}^{a_{3}}} x_{1}^{\sigma_{1}} x_{2}^{\sigma_{2}}, \frac{x_{2}^{a_{2}}}{x_{3}^{a_{3}}}$ corresponds to a middle polynomial and $\sigma_{1} \leq b_{1}-1, \sigma_{2} \leq b_{2}-1, b_{1}, b_{2}$ defined in Remark 3.2.

II. $m^{*}=x_{0}^{\sigma_{0}} x_{3}^{\sigma_{3}} \frac{x_{2}^{a_{2}}}{x_{3}^{a_{3}}} x_{1}^{\sigma_{1}} x_{2}^{\sigma_{2}}, \frac{x_{2}^{a_{2}}}{x_{3}^{a_{3}}}$ corresponds to an end polynomial and $\sigma_{1} \leq b_{1}-1, \sigma_{2} \leq c_{2}-1, b_{1}, c_{2}$ defined in Remark 3.2.

We will deal with the two cases separately.

Lemma 3.1. Let $m^{*}$ be as in I. above. Then $\sigma_{0}=a_{0}-1, \sigma_{3}=a_{3}-1, \sigma_{1}=b_{1}$ $-1, \sigma_{2}=b_{2}-1$.

Proof. Clearly $\sigma_{0} \leq a_{0}-1$ and $\sigma_{3} \leq a_{3}-1$. Suppose $\sigma_{3}<a_{3}-1$ or $0<a_{3}$ $-\sigma_{3}-1$. Then $\frac{x_{0}^{\sigma_{0}} x_{2}^{a_{2}+\sigma_{2}} x_{1}^{\sigma_{1}}}{x_{3}^{a_{3}-\sigma_{3}-1}}=0$, thus there must exist a monomial of a binomial in $\mathscr{B}_{2}$ dividing $x_{0}^{\sigma_{0}} x_{2}^{a_{2}+\sigma_{2}}$ or a monomial of a binomial in $\mathscr{B}_{3}$ dividing $x_{2}^{a_{2}+\sigma_{2}} x_{1}^{\sigma_{1}}$. In the first case since $\sigma_{0} \leq a_{0}-1$, this would produce an $x_{3}$-exponent $>a_{3}$ (since the $x_{3}$-exponents are monotonically increasing with decreasing $x_{0}$-exponents), thus $m^{*}=0$, which it is not. Hence this is not possible. In the second case, since $\sigma_{1}$ $\leq b_{1}-1$, by the algorithm in [BR], again the $x_{3}$-exponent produced is $>a_{3}$, thus $m^{*}=0$, a contradiction. Hence $\sigma_{3}=a_{3}-1$. Changing to the representation $\frac{x_{1}^{a_{1}}}{x_{0}^{a_{0}}}$, we obtain $\sigma_{0}=a_{0}-1$ by an analogous argument. We consider next $x_{0}^{a_{0}-1} \frac{x_{2}^{a_{2}}}{x_{3}}$ $x_{1}^{\sigma_{1}} x_{2}^{\sigma_{2}}, \sigma_{1} \leq b_{1}-1, \sigma_{2} \leq b_{2}-1$. Then by the algorithm of [BR] and the middle polynomial assumption, there does not exist a binomial in $\mathscr{B}_{3}$ with monomial term dividing $x_{1}^{\sigma_{1}} x_{2}^{\sigma_{2}+a_{2}+1}$, from which $\sigma_{2}=b_{2}-1$. By an analogous argument with the representation $\frac{x_{1}^{a_{1}}}{x_{0}^{a_{0}}}, \sigma_{1}=b_{1}-1$.

For the next three lemmata $\frac{x_{2}^{a_{2}}}{x_{3}^{a_{3}}}$ will correspond to an end polynomial.

Lemma 3.2. Let $\beta_{2}=x_{0}^{a_{0}} x_{2}^{a_{2}}-x_{1}^{a_{1}} x_{3}^{a_{3}} \in \mathscr{B}_{2}$ be an end polynomial. If $B\left(\beta_{2}\right) \neq$ $\emptyset$ let $f_{1}=x_{0}^{b_{0}} x_{3}^{b_{3}}-x_{1}^{b_{1}} x_{2}^{b_{2}} \neq f_{l}=x_{0}^{c_{0}} x_{3}^{c_{3}}-x_{1}^{c_{1}} x_{2}^{c_{2}}$ as defined in Remark 3.2. If $B\left(\beta_{2}\right)=\emptyset$ (i.e. if $\beta_{2}$ is the first or last polynomial in $\mathscr{B}_{2}$ and the algorithm in $[B R]$ proceeds such that $\left.B\left(\beta_{2}\right)=\emptyset\right)$, then let $f_{1}=f_{l}$ be either the first or last polynomial in $\mathscr{B}_{3}$ (depending upon if $\beta_{2}$ is the first or last polynomial in $\mathscr{B}_{2}$ ). Then $m_{1}=x_{0}^{a_{0}-1} x_{3}^{a_{3}-1}$. 
$\frac{x_{2}^{a_{2}}}{x_{3}^{a_{3}}} x_{1}^{b_{1}-1} x_{2}^{b_{2}-1}$ and $m_{l}=x_{0}^{a_{0}-1} x_{3}^{a_{3}-1} \frac{x_{2}^{a_{2}}}{x_{3}^{a_{3}}} x_{1}^{c_{1}-1} x_{2}^{c_{2}-1}$ are maximal multiples of $\frac{x_{2}^{a_{2}}}{x_{3}^{a_{3}}}$.

Proof. Since there does not exist $\beta_{i} \in \mathscr{B}_{i}, i=1,2$, with one of its monomial terms dividing $x_{0}^{a_{0}-1} x_{2}^{a_{2}+c_{2}-1}$ or $x_{1}^{c_{1}-1} x_{2}^{a_{2}+c_{2}-1}, x_{0}^{a_{0}-1} \frac{x_{2}^{a_{2}}}{x_{3}} x_{1}^{c_{1}-1} x_{2}^{c_{2}-1}=x_{3}^{a_{3}-1} \frac{x_{1}^{a_{1}}}{x_{0}}$. $x_{1}^{c_{1}-1} x_{2}^{c_{2}-1} \neq 0$ in $H_{m}^{1}(\bar{R})$. Clearly $x_{0}$ and $x_{3}$ annihilate this multiple. That this is also the case for $x_{1}$ and $x_{2}$ follows from $x_{0}^{c_{0}} x_{3}^{c_{3}}-x_{1}^{c_{1}} x_{2}^{c_{2}} \in \mathscr{B}_{3}$. The proof for the other multiple is analogous.

LEMMA 3.3. Let $m^{*}=x_{0}^{\sigma_{0}} x_{3}^{\sigma_{3}} \frac{x_{2}^{a_{2}}}{x_{3}^{a_{3}}} x_{1}^{\sigma_{1}} x_{2}^{\sigma_{2}}$ be as in II., i.e. $\frac{x_{2}^{a_{2}}}{x_{3}^{a_{3}}}$ corresponds to an end polynomial, $\sigma_{1} \leq b_{1}-1, \sigma_{2} \leq c_{2}-1, b_{1}, c_{2}$ as in Remark 3.2. Then $\sigma_{0}=a_{0}-$ 1 and $\sigma_{3}=a_{3}-1$.

Proof. As always $\sigma_{0} \leq a_{0}-1$ and $\sigma_{3} \leq a_{3}-1$. Suppose $\sigma_{3}<a_{3}-1$ or $0<a_{3}-\sigma_{3}-1$. Then $x_{0}^{\sigma_{0}} \frac{x_{2}^{a_{2}+\sigma_{2}}}{x_{3}^{a_{3}-\sigma_{3}-1}} x_{1}^{\sigma_{1}}=0$. Since $\sigma_{1} \leq b_{1}-1$, if $x_{2}^{a_{2}+\sigma_{2}} x_{1}^{\sigma_{1}}$ is to be divisible by a monomial term of a binomial in $\mathscr{B}_{3}$, its $x_{3}$-exponent is $>a_{3}$. But then $m^{*}=0$, a contradiction. If $x_{0}^{\sigma_{0}} x_{2}^{a_{2}+\sigma_{2}}$ is to be divisible by a monomial term of a binomial in $\mathscr{B}_{2}$, then since $\sigma_{0} \leq a_{0}-1$, its $x_{3}$-exponent again is $>a_{3}$. Thus again $m^{*}=0$, a contradiction. Hence $\sigma_{3}=a_{3}-1$. By replacing the representation $\frac{x_{2}^{a_{2}}}{x_{3}^{a_{3}}}$ by $\frac{x_{1}^{a_{1}}}{x_{0}^{a_{0}}}$ and an analogous argument, we obtain $\sigma_{0}=a_{0}-1$.

Lemma 3.4. Let $\frac{x_{2}^{a_{2}}}{x_{3}^{a_{3}}}$ correspond to an end polynomial $\beta_{2}=x_{0}^{a_{0}} x_{2}^{a_{2}}-x_{1}^{a_{1}} x_{3}^{a_{3}}$. Let $m=x_{0}^{a_{0}-1} x_{3}^{a_{3}-1} \frac{x_{2}^{a_{2}}}{x_{3}^{a_{3}}} x_{1}^{\sigma_{1}} x_{2}^{\sigma_{2}}$ be a maximal multiple of largest possible degree. (Note that the degree of $m$ here need not necessarily be $e\left(H_{\underline{m}}^{1}(\bar{R})\right)$. If $a_{1}>a_{2}$, then $\sigma_{1}=b_{1}-1$, $\sigma_{2}=b_{2}-1$, if $a_{1}<a_{2}$, then $\sigma_{1}=c_{1}-1, \sigma_{2}=c_{2}-1$ and if $a_{1}=a_{2}$, then either of the preceding two choices is possible.

Proof. Let $m=x_{0}^{a_{0}-1} \frac{x_{2}^{a_{2}+\sigma_{2}}}{x_{3}} x_{1}^{\sigma_{1}}$. We first determine the possibilities for $\sigma_{1}$ and $\sigma_{2}$. We consider two cases:

a) No monomial term of a binomial in $\mathscr{B}_{3}$ divides $x_{2}^{a_{2}+\sigma_{2}+1} x_{1}^{\sigma_{1}}$. Since $a_{0}-1$ $<a_{0}$ and $a_{2}+\sigma_{2}+1>a_{2}$, there must exist $x_{0}^{a_{0}-c_{0}} x_{2}^{a_{2}+c_{2}}-x_{1}^{a_{1}-c_{1}} x_{3}^{a_{3}+c_{3}} \in \mathscr{B}_{2}$, 
$x_{0}^{c_{0}} x_{3}^{c_{3}}-x_{1}^{c_{1}} x_{2}^{c_{2}} \in \mathscr{B}_{3}$, and $a_{2}+\sigma_{2}+1=a_{2}+c_{2}$, where $c_{i}, 0 \leq i \leq 3$ are as defined in Remark 3.2. Hence $\sigma_{2}=c_{2}-1$. By Lemma $3.2 \sigma_{1}=c_{1}-1$ now follows immediately.

b) There are monomial terms of binomials in $\mathscr{B}_{3}$, which divide $x_{2}^{a_{2}+\sigma_{2}+1} x_{1}^{\sigma_{1}}$. Since $m$ is a maximal multiple and since the $x_{0}$ and $x_{1}$ variables do not appear in any monomial of $\mathscr{B}\left(n_{1}, n_{2}, n_{3}\right)$, we also must have that $x_{2}^{a_{2}+\sigma_{2}} x_{1}^{\sigma_{1}+1}$ is divisible by a monomial term of a binomial in $\mathscr{B}_{3}$. For $B\left(\beta_{2}\right)=\emptyset$, we revert back to case a). Let $\emptyset \neq B\left(\beta_{2}\right)=\left\{x_{0}^{b_{0}} x_{3}^{b_{3}}-x_{1}^{b_{1}} x_{2}^{b_{2}}, \ldots, x_{0}^{c_{0}+a_{0}} x_{3}^{c_{3}-a_{3}}-x_{1}^{c_{1}+a_{1}} x_{2}^{c_{2}-a_{2}}, x_{0}^{c_{0}} x_{3}^{c_{3}}-x_{1}^{c_{1}} x_{2}^{c_{2}}\right\}$. In order for $x_{2}^{a_{2}+\sigma_{2}} x_{1}^{\sigma_{1}}$ to be as required, $\sigma_{1}$ has to be by one less than the $x_{1}$-exponent of an element in $\mathscr{B}_{3}$ and $a_{2}+\sigma_{2}$ by one less than the $x_{2}$-exponent of its successor. From this we get $a_{2}+\sigma_{2}<c_{2}$ (otherwise $m=0$ ), and by symmetry $a_{1}+\sigma_{1}<b_{1}$. Thus the maximal multiples to consider are

$$
\begin{aligned}
& m_{1}=x_{0}^{a_{0}-1} x_{3}^{a_{3}-1} \frac{x_{2}^{a_{2}}}{x_{3}^{a_{3}}} x_{1}^{b_{1}-1} x_{2}^{b_{2}-1}, \\
& m_{2}=x_{0}^{a_{0}-1} x_{3}^{a_{3}-1} \frac{x_{2}^{a_{2}}}{x_{3}^{a_{3}}} x_{1}^{\left(b_{1}-1\right)-a_{1}} x_{2}^{\left(b_{2}-1\right)+a_{2}}, \ldots, m_{l-1}=x_{0}^{a_{0}-1} x_{3}^{a_{3}-1} \frac{x_{2}^{a_{2}}}{x_{3}^{a_{3}}} x_{1}^{\left(c_{1}-1\right)+a_{1}} x_{2}^{\left(c_{2}-1\right)-a_{2}} .
\end{aligned}
$$

The statement about the degree now is immediate.

COROLlary 3.1. There exists an element of maximal degree amongst the maximal multiples of Lemma 3.1 and Lemma 3.2.

Proof. This follows from the preceding, the fact that the initial maximal multiple $m=x_{0}^{\gamma_{0}} x_{3}^{\gamma_{3}} \frac{x_{2}^{a_{2}}}{x_{3}^{a_{3}}} x_{1}^{\gamma_{1}} x_{2}^{\gamma_{2}}$ was arbitrary and only homogeneous polynomials were used in all of the above (thus no change in degree), and by the structure theorem (Theorem 2.1) in Section 2.

Lemma 3.5. Assume that $m_{1}$ and $m_{2}$ are maximal multiples as in Lemma 3.1 and Lemma 3.2 which correspond to succesive binomials $b_{1}=x_{0}^{a_{0}} x_{2}^{a_{2}}-$ $x_{1}^{a_{1}} x_{3}^{a_{3}}$ and $b\left(b_{1}\right)=b_{2}, b=x_{0}^{d_{0}} x_{3}^{d_{3}}-x_{1}^{d_{1}} x_{2}^{d_{2}}$. Then $d\left(m_{2}\right)-d\left(m_{1}\right)=\Delta=d_{2}-d_{0}$.

Proof. The degree difference between $m_{1}=x_{0}^{a_{0}-1} x_{3}^{a_{3}-1} \frac{x_{2}^{a_{2}}}{x_{3}^{a_{3}}} x_{1}^{d_{1}-1} x_{2}^{d_{2}-1}$ and $m_{2}$ $=x_{0}^{a_{0}-d_{0}-1} x_{3}^{a_{3}+d_{3}-1} \frac{x_{2}^{a_{2}+d_{2}}}{x_{3}^{a_{3}+d_{3}}} x_{1}^{d_{1}-1} x_{2}^{d_{2}-1}$ is $d_{2}-d_{1}$. 
THEOREM 3.1. For a maximal multiple of maximal degree only the maximal multiples in Lemma 3.2 need be considered. If $\frac{x_{2}^{a_{2}}}{x_{3}^{a_{3}}}$ corresponds to the end polynomial $x_{0}^{a_{0}} x_{2}^{a_{2}}-x_{1}^{a_{1}} x_{3}^{a_{3}}$ and $a_{1}>a_{2}$ then $m_{1}$ of Lemma 3.2 needs to be considered, if $a_{1}<a_{2}$ then $m_{l}$ of Lemma 3.2 will do and if $a_{1}=a_{2}$, then $d\left(m_{1}\right)=d\left(m_{l}\right)$.

Proof. This follows from Corollary 3.1, Lemma 3.5 and Lemma 3.4.

Remark 3.3. (i) It follows readily that an element of minimal degree in $H_{\underline{m}}^{1}(\bar{R})$ is also obtained from an element $\frac{x_{2}^{a_{2}}}{x_{3}^{a_{3}}}$, which corresponds to an end polynomial in $\mathscr{B}_{2}$.

(ii) We note that our algorithmic procedure in this section calculates also the socle of $H_{\underline{m}}^{1}(\bar{R})$.

We conclude this section with an algorithmic computation of $k\left(C\left(n_{1}, n_{2}, n_{3}\right)\right)$.

1. Let $\alpha_{i}$ be minimal positive integers such that $\alpha_{i} n_{i}=\alpha_{i j} n_{j}+a_{i k} n_{k} \in$ $\left\langle n_{j}, n_{k}\right\rangle,\{i, j, k\}=\{1,2,3\}$. These equations define polynomials

$$
f_{1}=x_{1}^{\alpha_{1}}-x_{2}^{\alpha_{12}} x_{3}^{\alpha_{13}}, \quad f_{2}=x_{2}^{\alpha_{2}}-x_{1}^{\alpha_{21}} x_{3}^{\alpha_{23}}, \quad f_{3}=x_{3}^{\alpha_{3}}-x_{1}^{\alpha_{31}} x_{2}^{\alpha_{32}} .
$$

Assume that $0<\alpha_{2}<\alpha_{21}+\alpha_{23}, \alpha_{21}>0$, and $0<\alpha_{3}<\alpha_{31}+\alpha_{32}$, with $\alpha_{31}>0$. (If one of these conditions is not satisfied, then $C\left(n_{1}, n_{2}, n_{3}\right)$ is Cohen-Macaulay.) Let $\{i, j\}=\{2,3\}$ and $\alpha_{i 1} \leq \alpha_{j 1}$. Then from $f_{2}$ and $f_{3}$ one obtains either $f_{2}\left(f_{3}\right)$ or $f_{3}\left(f_{2}\right)$ equal to:

$$
f_{12}=x_{j}^{\alpha_{j}+\alpha_{i j}}-x_{1}^{\alpha_{j 1}-\alpha_{i 1}} x_{i}^{\alpha_{j i}+\alpha_{i}} .
$$

We repeat the process as in the Euclidean algorithm for $\alpha_{21}$ and $\alpha_{31}$ until we obtain a polynomial $x_{2}^{\beta_{2}}-x_{1}^{\beta_{21}} x_{3}^{\beta_{23}}$ with $\beta_{2} \geq \beta_{21}+\beta_{23}$.

Assume $f_{2} \neq-f_{1}$ and $f_{3} \neq-f_{1}$. Then we obtain:

$$
\begin{gathered}
\mathscr{B}^{*}=\left\{f_{1}, h_{1} \in\left\{f_{2}, f_{3}\right\}, f_{11} \in\left\{f_{2}, f_{3}\right\}, f_{12}, \ldots, f_{1 l_{1}}=h_{2} ; \ldots ; f_{k 1}, \ldots,\right. \\
\left.f_{k, l_{k}}=h_{k+1} ; \ldots ; f_{r-1,1}, \ldots, f_{r-1, l_{r-1}}=h_{r} ; f_{r 1}, \ldots, f_{r l_{r}}\right\},
\end{gathered}
$$

where $h_{1}\left(f_{11}\right)=f_{12}, \ldots, h_{1}\left(f_{1, l_{1}-1}\right)=h_{2}, h_{2}\left(h_{1}\right)=f_{21}, \ldots$

If $f_{2}=-f_{1}$ or $f_{3}=-f_{1}$ we delete $f_{2}$ or $f_{3}$ and obtain:

$$
\begin{gathered}
\mathscr{B}^{*}=\left\{f_{1}, h_{1} \in\left\{f_{2}, f_{3}\right\}, f_{11}, \ldots, f_{1 e_{1}}=h_{2} ; \ldots ; f_{r-1,1}, \ldots,\right. \\
\left.f_{r-1, e_{r-1}}=h_{r} ; f_{r 1}, \ldots, f_{r e_{r}}\right\},
\end{gathered}
$$


where $h_{1}\left(-f_{1}\right)=f_{11}, \ldots, h_{1}\left(f_{1, e_{1}-1}\right)=h_{2}, h_{2}\left(h_{1}\right)=f_{21}, \ldots$

In both cases, with the homogenized binomials of $\mathscr{B}^{*}$ denoted by capital letters and with corresponding subscripts, we have:

$$
\begin{aligned}
& \mathscr{B}\left(n_{1}, n_{2}, n_{3}\right)=\left\{F_{1}, H_{1}, F_{11}, \ldots, F_{1 n_{1}}=H_{2} ; \ldots ; F_{k 1}, \ldots, F_{k, n_{k}}=H_{k+1} ; \ldots ;\right. \\
& \left.F_{r-1,1}, \ldots, F_{r-1, n_{r-1}}=H_{r} ; F_{r 1}, \ldots, F_{r n_{r}}\right\} \text {. }
\end{aligned}
$$

and

$$
\mathscr{B}_{2}=\left\{\begin{array}{l}
\left\{F_{11}, \ldots, F_{1 n_{1}} ; F_{31} ; \ldots, F_{3 n_{3}} ; \ldots ; F_{r 1}, \ldots, F_{r, n_{r}-1}\right\} \text { if } r \text { is odd } \\
\left\{H_{1}, F_{21}, \ldots, F_{2 n_{2}} ; F_{41}, \ldots, F_{4 n_{4}} ; \ldots ; F_{r 1}, \ldots, F_{r, n_{r}-1}\right\} \text { if } r \text { is even. }
\end{array}\right.
$$

2. Let $d_{0}=\operatorname{deg}\left(F_{11}\right), d_{1}=\operatorname{deg}\left(H_{1}\right), \ldots, d_{r}=\operatorname{deg}\left(H_{r}\right)$, and

$$
d_{r+1}=\left\{\begin{array}{l}
\operatorname{deg}\left(F_{r, n_{r}-1}\right) \text { if } n_{r}>1, \\
d_{r-1} \text { if } n_{r}=1
\end{array}\right.
$$

Let $\mathcal{M}=\max \left\{d_{i}+d_{i+1} ; i=0, \ldots, r\right\}$, and $m=\min \left\{a_{2}-a_{3} ; x_{0}^{a_{0}} x_{2}^{a_{2}}-x_{1}^{a_{1}} x_{3}^{a_{3}} \in\right.$ $\left.\mathscr{B}_{2}\right\}$.

Then $k\left(C\left(n_{1}, n_{2}, n_{3}\right)\right)=M-m-3$.

\section{Castelnuovo-Mumford regularity for monomial curves in $P_{K}^{3}$}

Let $A=\bigoplus_{i \geq 0} A_{i}$ be a Noetherian graded standard $K$-algebra, i.e. $A=R / I$, where $I$ is a homogeneous ideal of a polynomial ring $R=K\left[x_{0}, \ldots, x_{n}\right]$ in $n+1$ indeterminates. Let $\underline{m}=\bigoplus_{i>0} A_{i}$ be the homogeneous maximal ideal of $A$. Recall that the Castelnuovo-Mumford regularity of $A$ is defined as follows:

$$
\operatorname{reg} A=\max \left\{e\left(H_{\underline{m}}^{i}(A)+i ; i \leq \operatorname{dim} A\right\},\right.
$$

where $e\left(H_{\underline{m}}^{i}(A)\right)=\max \left\{j ;\left[H_{\underline{m}}^{i}(A)\right]_{j} \neq 0\right\}$. This is an important invariant of $A$. If $A$ has the following minimal graded free resolution:

$$
0 \rightarrow \bigoplus_{i=1}^{n_{p}} R\left(-e_{p i}\right) \rightarrow \cdots \rightarrow \bigoplus_{i=1}^{n_{1}} R\left(-e_{1 i}\right) \rightarrow R \rightarrow A \rightarrow 0,
$$

it is well-known that (see $[M],[E G])$

$$
\operatorname{reg} A=\max \left\{e_{j i}-j ; j=1, \ldots, p \text { and } 1 \leq i \leq n_{j}\right\} .
$$

In this section we will prove:

THEorem 4.1. Assume that $C=C\left(n_{1}, n_{2}, n_{3}\right)$ is not Cohen-Macaulay $(C-M)$. Then 


$$
\operatorname{reg}(K[S])=e\left(H_{\underline{m}}^{1}(K[S])\right)+1
$$

As an immediate consequence of this theorem and Theorem 2.1 we have

Corollary 4.1. If $C\left(n_{1}, n_{2}, n_{3}\right)$ is not $C-M$, then

$$
k\left(C\left(n_{1}, n_{2}, n_{3}\right)\right)=\operatorname{reg}(K[S])-a\left(H_{\underline{m}}^{1}(K[S])\right),
$$

where $a\left(H_{\underline{m}}^{1}(K[S])\right)$ is defined in Section 1 .

The proof of Theorem 4.1 is divided into several lemmata. First, as in Section 1 , for $i=0,1$, the set $A_{i}\left(S_{i}, n_{3}\right)=\left\{0, \omega_{i}(1), \ldots, \omega_{i}\left(n_{3}-1\right)\right\}$, where

$$
\begin{gathered}
\omega_{i}(j)=\min \left\{a \in S_{i} ; a \equiv j \bmod n_{3}\right\}, j=1, \ldots, n_{3}-1, \\
H \cap\left(A_{0}\left(S_{0}, n_{3}\right) \times A_{1}\left(S_{1}, n_{3}\right)\right)=\left\{\left(\omega_{0}(i), \omega_{1}\left(n_{3}-i\right)\right) ; i=0, \ldots, n_{3}-1\right\}=\tilde{I},
\end{gathered}
$$
and

$$
J=\tilde{I} \backslash S .
$$

It is clear that if $\omega_{1}(i)=a n_{1}+b n_{2}$ then $\omega_{0}\left(n_{3}-i\right) \leq a\left(n_{3}-n_{1}\right)+b\left(n_{3}-\right.$ $\left.n_{2}\right)$ and the equality holds if and only if $a\left(n_{3}-n_{1}\right)+b\left(n_{3}-n_{2}\right) \notin n_{3}+S_{0}$. Note that $J$ generates $K\left[S^{\prime} \backslash S\right]$ as $K[S]$-module (see [FH], Lemma 2.4). $C\left(n_{1}, n_{2}, n_{3}\right)$ is not $\mathrm{C}-\mathrm{M}$ if and only if $J \neq \emptyset$.

Next we define $\alpha_{1}$ (resp. $\left.\beta_{1}\right)$ to be the least number such that $\delta_{1}\left(\alpha_{1} n_{1}\right)<\alpha_{1}$ (resp. $\beta_{1} n_{2} \in n_{3}+S_{1}$ ). Analogously we define the corresponding numbers $\alpha_{0}, \beta_{0}$ for the generators $n_{3}-n_{2}<n_{3}-n_{1}<n_{3}$ of $S_{0}$.

Remark 4.1. From the definition of $\alpha_{i}, \beta_{i}, i=0,1$, we get $\beta_{0} \leq \alpha_{1}$ and $\beta_{1}$ $\leq \alpha_{0}$. These relations are also immediate consequences of the algorithm in [BR].

In the next lemma we use the following partial order $\leq$ on the set $\mathbf{N}^{2}:(a, b)$ $\leq\left(a^{\prime}, b^{\prime}\right)$ if $a \leq a^{\prime}$ and $b \leq b^{\prime}$.

Lemma 4.1. If the set $\left\{(a, b) ; 0 \leq a<\alpha_{1}, 0<b \leq \beta_{1}\right.$ and $a n_{1}+b n_{2}=$ $m n_{3}$ for some $\left.m>0\right\} \neq \emptyset$ then it has a unique minimal element.

Proof. Assume that there are two equations: $m_{1}^{\prime} n_{1}+m_{2}^{\prime} n_{2}=m^{\prime} n_{3}$ and $m_{1} n_{1}$ $+m_{2} n_{2}=m n_{3}$ with $m_{1}^{\prime}>m_{1}$ and $m_{2}^{\prime}<m_{2}$ or $m_{1}^{\prime}<m_{1}$ and $m_{2}^{\prime}>m_{2}$. Subtracting one equation from the other we get $a n_{1}=b n_{2}+c n_{3}$ or $b n_{2}=a n_{1}+c n_{3}$, where $0 \leq a<\alpha_{1}, 0 \leq b \leq \beta_{1}$. By the minimality of $\alpha_{1}$ and $\beta_{1}$, this can happen only if 
$b=\beta_{1}$, i.e. one of the two numbers $m_{2}$ and $m_{2}^{\prime}$ is 0 . But this contradicts the minimality of $\alpha_{1}$.

If the set defined in Lemma 4.1 is not empty, we denote its minimal element by $\left(m_{1}, m_{2}\right)$. Otherwise we set $m_{1}=\alpha_{1}$ and $m_{2}=\beta_{1}$. Let us introduce the following set:

$$
B_{1}=\left\{(a, b) ; 0 \leq a<m_{1}, 0 \leq b<\beta_{1}\right\} \cup\left\{(a, b) ; m_{1} \leq a<\alpha_{1}, 0 \leq b<m_{2}\right\} .
$$

Then we have:

Lemma 4.2. The set $B_{1}^{\prime}=\left\{a n_{1}+b n_{2} ;(a, b) \in B_{1}\right\}$ consists of distinct elements and coincides with the Apery sequence $A_{1}\left(S_{1}, n_{3}\right)$ of $S_{1}$ (up to permutations). Moreover, for each element $a n_{1}+b n_{2} \in B_{1}^{\prime}$ we have $\delta_{1}\left(a n_{1}+b n_{2}\right)=a+b$.

Proof. Since $A_{1}\left(S_{1}, n_{3}\right) \subseteq\left(\mathbf{N} n_{1}+\mathbf{N} n_{2}\right) \backslash\left(n_{3}+S_{1}\right)$, we must have $A_{1}\left(S_{1}, n_{3}\right)$ $\subseteq B_{1}^{\prime}$. Let $(a, b) \in B_{1}$. Choose an element $a^{\prime} n_{1}+b^{\prime} n_{2} \in A_{1}\left(S_{1}, n_{3}\right)$ such that $\left(a^{\prime}, b^{\prime}\right) \in B_{1}$ and $a n_{1}+b n_{2}=c n_{3}+a^{\prime} n_{1}+b^{\prime} n_{2}$. From the minimality of $\alpha_{1}$ and $\beta_{1}$ it follows that $a \geq a^{\prime}, b \geq b^{\prime}$. Then we have $\left(a-a^{\prime}\right) n_{1}+\left(b-b^{\prime}\right) n_{2}=c n_{3}$. By Lemma 4.1, $\left(a-a^{\prime}, b-b^{\prime}\right) \geq\left(m_{1}, m_{2}\right)$ unless $a-a^{\prime}=b-b^{\prime}=c=0$. Since $a<m_{1}$ or $b<m_{2}$, we must have $a=a^{\prime}, b=b^{\prime}$ and $c=0$. This proves that the element $a n_{1}+b n_{2} \notin\left(n_{3}+S_{1}\right)$, i.e. $B_{1}^{\prime}=A_{1}\left(S_{1}, n_{3}\right)$ and the elements in $B_{1}^{\prime}$ are distinct. From this it also follows that if $a n_{1}+b n_{2}=c n_{3}+a^{\prime} n_{1}+b^{\prime} n_{2}$ for some $c, a^{\prime}, b^{\prime} \in \mathbf{N}$, then $c=0$ and $a+b \leq a^{\prime}+b^{\prime}$. (Note that $a+b<a^{\prime}+$ $b^{\prime}$ is possible.) Hence $\delta_{1}\left(a n_{1}+b n_{2}\right)=a+b$, as required.

Remark 4.2. An equivalent formula to the one in Lemma 4.2 was given by Rödseth (see [Ro], pp. 175).

Lemma 4.3. Assume that $(a, b) \in B_{1}$ and $a<\beta_{0}$. Then $a\left(n_{3}-n_{1}\right)+b\left(n_{3}-\right.$ $\left.n_{2}\right) \in A_{0}\left(S_{0}, n_{3}\right)$.

Proof. Assume that $a\left(n_{3}-n_{1}\right)+b\left(n_{3}-n_{2}\right)=a^{\prime}\left(n_{3}-n_{1}\right)+b^{\prime}\left(n_{3}-n_{2}\right)$ $+c^{\prime} n_{3}$, where $c^{\prime}>0$ and $a^{\prime}, b^{\prime} \in \mathbf{N}$. By Remark 2.1 (or [BR]), $b<\beta_{1} \leq \alpha_{0}$, and by assumption $a<\beta_{0}$. From the minimality of $\alpha_{0}$ and $\beta_{0}$ it follows that $(a, b) \geq$ $\left(a^{\prime}, b^{\prime}\right)$. We then get: $\left(a-a^{\prime}\right) n_{1}+\left(b-b^{\prime}\right) n_{2}=\left(a-a^{\prime}+b-b^{\prime}-c^{\prime}\right) n_{3}$. By Lemma 4.1 we must have $\left(a-a^{\prime}, b-b^{\prime}\right) \geq\left(m_{1}, m_{2}\right)$ which implies that $(a, b) \geq\left(m_{1}, m_{2}\right)$, a contradiction. 
Lemma 4.4. If $C\left(n_{1}, n_{2}, n_{3}\right)$ is not $C-M$, then

$$
\max \left\{\delta_{1}(a) ; a \in A_{1}\left(S_{1}, n_{3}\right)\right\} \leq \max \left\{\delta_{0}\left(e_{0}\right), \delta_{1}\left(e_{1}\right) ;\left(e_{0}, e_{1}\right) \in J\right\} .
$$

Proof. First assume that $\beta_{0}=\alpha_{1}$. Let $e=\left(\omega_{0}\left(n_{3}-i\right), \omega_{1}(i)\right)$ be an arbitrary element of $\tilde{I}$. We write $\omega_{1}(i)=a n_{1}+b n_{2}$ with $(a, b) \in B_{1}$. By Lemma 4.3, $a\left(n_{3}-n_{1}\right)+b\left(n_{3}-n_{2}\right) \in A_{0}\left(S_{0}, n_{3}\right)$. Hence $\omega_{0}\left(n_{3}-i\right)=a\left(n_{3}-n_{1}\right)+b\left(n_{3}-n_{2}\right)$, which implies that $e \in S$. This shows that $\tilde{I} \subset S$, i.e. $C\left(n_{1}, n_{2}, n_{3}\right)$ is C-M, a contradiction. So, from Remark 4.1, we must have $\beta_{0}<\alpha_{1}$, and, by symmetry, $\beta_{1}<\alpha_{0}$.

Now we consider two cases separately, noting that $\delta(i, j)$ equals the number of steps between $-i$ and $j$ in the congruence class $n_{3}$.

Case 1: $\left(m_{1}, m_{2}\right)=\left(\alpha_{1}, \beta_{1}\right)$.

By Lemma 4.2 there exists $j$ such that $\omega_{1}(j)=\left(\alpha_{1}-1\right) n_{1}+\left(\beta_{1}-1\right) n_{2} \in A_{1}\left(S_{1}\right.$, $n_{3}$ ). Since $\alpha_{1}-1 \geq \beta_{0}$,

$$
\left(\alpha_{1}-1\right)\left(n_{3}-n_{1}\right)+\left(\beta_{1}-1\right)\left(n_{3}-n_{2}\right) \in n_{3}+S_{0} .
$$

By the definition of Apery sequence, we then get $\omega_{0}\left(n_{3}-j\right)<\left(\alpha_{1}-1\right)\left(n_{3}-n_{1}\right)$ $+\left(\beta_{1}-1\right)\left(n_{3}-n_{2}\right)$. Therefore $\delta\left(\left(\omega_{0}\left(n_{3}-j\right), \omega_{1}(j)\right)\right)<\delta\left(\left(\alpha_{1}-1\right)\left(n_{3}-n_{1}\right)\right.$ $\left.+\left(\beta_{1}-1\right)\left(n_{3}-n_{2}\right), \quad\left(\alpha_{1}-1\right) n_{1}+\left(\beta_{1}-1\right) n_{2}\right)=\left(\alpha_{1}-1\right)+\left(\beta_{1}-1\right)=\delta_{1}\left(\omega_{1}(j)\right)$. Thus by Lemma 2.1, $\left(\omega_{0}\left(n_{3}-j\right), \omega_{1}(j)\right) \notin S$. Hence

$$
\begin{aligned}
\max \left\{\delta_{1}(a) ; a \in A_{1}\left(S_{1}, n_{3}\right)\right\} \leq & \alpha_{1}-1+\beta_{1}-1= \\
& \delta_{1}\left(\omega_{1}(j)\right) \leq \max \left\{\delta_{1}\left(e_{1}\right) ;\left(e_{0}, e_{1}\right) \in J\right\} .
\end{aligned}
$$

Case 2: $m_{1}<\alpha_{1}$ and $m_{2} \leq \beta_{1}$.

Similarly as in Case $1,\left(\alpha_{1}-1\right) n_{1}+\left(m_{2}-1\right) n_{2}$ is the second component of an element of $J$. Hence

$$
\max \left\{\delta_{1}\left(e_{1}\right) ;\left(e_{0}, e_{1}\right) \in J\right\} \geq\left(\alpha_{1}-1\right)+\left(m_{2}-1\right) .
$$

If $m_{1}>\beta_{0}$ then considering $\left(m_{1}-1\right) n_{1}+\left(\beta_{1}-1\right) n_{2}$ we get similarly as above that

$$
\max \left\{\delta_{1}\left(e_{1}\right) ;\left(e_{0}, e_{1}\right) \in J\right\} \geq\left(m_{1}-1\right)+\left(\beta_{1}-1\right) .
$$

(1), (2) and the construction of $B_{1}$ imply the claim.

Finally let $m_{1} \leq \beta_{0}$. By Remark 2.1, $m_{2} \leq \beta_{1}<\alpha_{0}$. Note that $m_{1} n_{1}+m_{2} n_{2}=$ $m n_{3}(m>0)$ if and only if $m_{1}\left(n_{3}-n_{1}\right)+m_{2}\left(n_{3}-n_{2}\right)=m^{\prime} n_{3}\left(m^{\prime}=m_{1}+m_{2}\right.$ $m>0)$. This implies that in our case $\left(m_{2}, m_{1}\right)$ is just the unique minimal element defined by Lemma 4.1 for $n_{3}-n_{2}<n_{3}-n_{1}<n_{3}$. Applying Lemma 4.2 for the 
Apery sequence $A_{0}\left(S_{0}, n_{3}\right)$ we get that $A_{0}\left(S_{0}, n_{3}\right)$ is in one-to-one correspondence with the set

$$
B_{0}=\left\{(a, b) ; 0 \leq a<m_{2}, 0 \leq b<\beta_{0}\right\} \cup\left\{(a, b) ; m_{2} \leq a<\alpha_{0}, 0 \leq b<m_{1}\right\} .
$$

Since $\beta_{1}<\alpha_{0}$, similarly as in Case 1 we can show that $\left(\alpha_{0}-1\right)\left(n_{3}-n_{2}\right)+$ $\left(m_{1}-1\right)\left(n_{3}-n_{1}\right)$ is the first component of an element of $J$. Hence

$$
\max \left\{\delta_{0}\left(e_{0}\right) ;\left(e_{0}, e_{1}\right) \in J\right\} \geq \alpha_{0}-1+m_{1}-1>\beta_{1}+m_{1}-2 .
$$

Combining (1) and (3) we get

$$
\begin{aligned}
& \max \left\{\delta_{0}\left(e_{0}\right), \delta_{1}\left(e_{1}\right) ;\left(e_{0}, e_{1}\right) \in J\right\} \geq \max \left\{\alpha_{1}+m_{2}-2, m_{1}+\beta_{1}-2\right\} \\
&=\max \left\{\delta_{1}(a) ; a \in B_{1}^{\prime}\right\}=\max \left\{\delta_{1}(a) ; a \in A_{1}\left(S_{1}, n_{3}\right)\right\}, \text { as required }
\end{aligned}
$$

Now we are able to prove Theorem 4.1 .

Proof of Theorem 4.1. It suffices to prove that $e\left(H_{\underline{m}}^{2}(K[S])\right) \leq e\left(H_{\underline{m}}^{1}(K[S])\right.$ - 1. By [TH], Corollary 3.8, $H_{\underline{m}}^{2}(K[S]) \cong K\left[\mathbf{Z}(S) \backslash S_{0} \cup \underline{\cup} S_{1}\right]$, where $\mathbf{Z}(S)$ is the additive subgroup generated by $S$. Hence

$$
\left.e\left(H_{\underline{m}}^{2}(K[S])\right)=\max \left\{\delta\left(\omega_{0}\left(n_{3}-i\right)-n_{3}, \omega_{1}(i)-n_{3}\right)\right) ; 0 \leq i<n_{3}\right\}=\max _{e \in \tilde{I}} \delta(e)-2 .
$$

(See also Proposition 1 and Corollary 5 in [BSS].) Let $\omega_{1}(i)=a n_{1}+b n_{2}$, where $(a, b) \in B_{1}$. Since $\omega_{0}\left(n_{3}-i\right) \leq a\left(n_{3}-n_{1}\right)+b\left(n_{3}-n_{2}\right)$, it follows that $\delta\left(\omega_{0}\left(n_{3}-\right.\right.$ $\left.i), \omega_{1}(i)\right) \leq \delta\left(a\left(n_{3}-n_{1}\right)+b\left(n_{3}-n_{2}\right), \omega_{1}(i)\right)=a+b=\delta_{1}\left(\omega_{1}(i)\right)$. Therefore

$$
e\left(H_{\underline{m}}^{2}(K[S])\right)=\max _{e \in \tilde{I}} \delta(e)-2 \leq \max _{a \in A_{1}\left(S_{1}, n_{3}\right)} \delta(a)-2 .
$$

On the other hand, since $H_{\underline{m}}^{1}(K[S]) \cong K\left[S^{\prime} \backslash S\right], e\left(H_{\underline{m}}^{1}(K[S])=\max _{e \in S^{\prime} \backslash S}\right.$ $\delta(e)$. Let $e=\left(e_{0}, e_{1}\right) \in S^{\prime} \backslash S$ be an arbitrary element. By Lemma 2.1 we get that $\delta_{0}\left(e_{0}\right)>\delta(e), \delta_{1}\left(e_{1}\right)>\delta(e)$ and $e+\left(\delta_{0}\left(e_{0}\right)-\delta(e)-1\right) t_{0} \in S^{\prime} \backslash S$, and $e+$ $\left(\delta_{1}\left(e_{1}\right)-\delta(e)-1\right) t_{3} \in S^{\prime} \backslash S$. As a consequence, $\max _{f \in S^{\prime} \backslash S} \delta(f) \geq \max \left\{\delta_{0}\left(e_{0}\right)\right.$, $\left.\delta_{1}\left(e_{1}\right)\right\}-1$. Hence

$$
e\left(H_{\underline{m}}^{1}(K[S])\right) \geq \max _{e \in J}\left\{\delta_{0}\left(e_{0}\right), \delta_{1}\left(e_{1}\right)\right\}-1 .
$$

Combining (4) (5) and Lemma 4.4 completes the proof of the theorem.

Analyzing the proof of Lemma 4.4 we get the following criterion for the Cohen-Macaulay property of $C\left(n_{1}, n_{2}, n_{3}\right)$. 
COROLlaRY 4.1. The following are equivalent:

(i) $C\left(n_{1}, n_{2}, n_{3}\right)$ is $C-M$,

(ii) $\alpha_{1}=\beta_{0}$,

(iii) $\alpha_{0}=\beta_{1}$.

Proof. We can use [B2] in order to give another proof as follows. By [B2] $C\left(n_{1}, n_{2}, n_{3}\right)$ is $\mathrm{C}-\mathrm{M}$ precisely when $\mu\left(I\left(C\left(n_{1}, n_{2}, n_{3}\right)\right)\right) \leq 3$, where $\mu$ denotes the minimum number of generators. Now from the algorithm in [BR] the equivalence of (i), (ii), and (iii) follows immediately.

In the next section we need the following.

Example 4.1. Let us consider the curve $C(1, a, d)$, i.e. $n_{1}=1, n_{2}=a$ and $n_{3}=d$. Let $d=p a+q$, where $0 \leq q<a$. Then $\beta_{1}=p+1$ if $q>0$ and $\beta_{1}=p$ if $q=0$. From the equation $\alpha_{0}(d-a)=y(d-1)+z d, y, z \in \mathbf{N}$, one can easily check that $\alpha_{0}=\beta_{1}$ if and only if $a<p+q+1$ or $q=0$. Hence $C(1, a, d)$ is $\mathrm{C}-\mathrm{M}$ if and only if $a<p+q+1$ or $q=0$.

\section{Monomial curves with at most one singular point}

In this section for notational convenience we denote $n_{2}$ by $a$ and $n_{3}$ by $d$. We will consider the class of curves $C=C(1, a, d)$ and we compute $k(C(1, a, d))$ explicitly. From this it will become apparent, that to calculate $k(C)$ for a class of curves still is a formidable task. We always assume that $C$ is not C-M. Let $d=p a+q$, where $0 \leq q<a$. By Example 4.1 our assumption on $C$ says that $a \geq p+q+1$ and $q>0$.

Lemma 5.1. Assume that $C(1, a, d)$ is not $C-M$. Then

(i) Every $S$-minimal element $e$ of $S^{\prime} \backslash S$ has the form $e=((\alpha+\beta p)(d-a)$, $\alpha a-\beta q)$ for some positive integers $\alpha \leq \beta$ such that $1<\alpha a-\beta q<a$. Moreover. $\delta(e)=\alpha+\beta(p-1)$.

(ii) $((p+1)(d-a), a-q)$ is an $S$-minimal element of $S^{\prime} \backslash S$.

(iii) $\left([\mathrm{FH}]\right.$, Lemma 5.3) $a\left(H_{m}^{1}(K[S])\right)=p$.

Proof. (i) From Lemma 2.2 we know that $e=(m(d-a), n)$ for some positive $m, n$ with $n<a$ and $n-m a=-l d$, where $l>0$. Hence $n=m a-l(p a+$ $q)=(m-l p) a-l q$. Setting $\alpha=m-l p$ and $\beta=l$ we can easily get the assertions. 
(ii) is immediate from Lemma 2.1 and the fact that $p+q+1 \leq a$.

(iii) Since $S$-minimal elements of $S^{\prime} \backslash S$ generate $K\left[S^{\prime} \backslash S\right]$ as $K[S]$-module, $a\left(H_{m}^{1}(K[S])\right)=\min \delta(e)$, where $e$ runs over the set of $S$-minimal elements of $S^{\prime} \backslash S$. Hence (iii) follows from (i) and (ii).

The main result of this section is the following:

Theorem 5.1. Assume that $C(1, a, d)$ is not $C-M$. Then $k(C(1, a, d))=$ $\operatorname{diam}(M(C))=a-2$.

The proof of this theorem is based on estimating degrees of elements in the numerical semigroup $S_{1}=\langle 1, a, d\rangle=\mathrm{N}$ with respect to the generators $1, a, d$. Namely we need the following technical lemma.

Lemma 5.2. Assume that $p, q \geq 1$ and $p+q+1 \leq a$. Then

$$
\delta_{1}((a-q)+l+n a) \leq a+p-2,
$$

for any nonnegative integers $l, n$ such that $l+n \leq a-2$.

Having this lemma we can prove the main Theorem 5.1 as follows: By Lemma 5.1, $e=((p+1)(d-a), a-q)$ is an $S^{\prime}$-minimal element of $S^{\prime} \backslash S$ and $\delta(e)=$ $p$. Let $f=k t_{0}+l t_{1}+n t_{2}+m t_{3}$ be an arbitrary element of $S$ with $\delta(f)=a-2$. We have $e+t_{3}=(p+1) t_{2} \in S$. Hence, if $m>0, e+f \in S$. If $m=0$, then the second component of $e+f$ is $[e+f]_{1}=a-q+l+n a$. By Lemma 5.2, $\delta_{1}\left([e+f]_{1}\right) \leq a+p-2=\delta(e)+\delta(f)=\delta(e+f)$. By Lemma 2.1 it follows that $e+f \in S$ too. Therefore $e+f \in S$ for any $f \in S$ with $\delta(f) \geq a-2$. Let $g$ be now an element of $S^{\prime} \backslash S$ having the maximal degree. $g$ is, of course, an $S$-maximal element of $S^{\prime} \backslash S$. By Proposition 2.3, $g=e+h$ for some element $h \in$ $S$. Since $g \notin S$, it follows from the above consideration that $\delta(h)<a-2$. Hence $\delta(g) \leq a+p-3$, and so $e\left(H_{\underline{m}}^{1}(K[S])\right) \leq a+p-3$. By Lemma 5.1 (iii) we then get $k(C(1, a, d)) \leq \operatorname{diam}(M(C)) \leq a-2$. On the other hand, by [FH], Lemma $5.2, k(C(1, a, d)) \geq a-2$. Hence $k(C(1, a, d))=\operatorname{diam}(M(C))=a-2$, as required.

From Theorem 5.1 and Theorem 4.1 we get

COROLlary 5.1. $\operatorname{reg}(K[S])=a+p-2$. 
The rest of this section is devoted to the proof of Lemma 5.2. The main idea is the following: Let $E=a-q+l+n a$. We consider various representations of $E$ and try to locate one with a small sum of coefficients. Here, under a representation of $E$ we mean a sum $E=x \cdot 1+y \cdot a+z \cdot d$ with nonnegative integral coefficients $x, y, z$.

We first define some nonnegative integers as follows:

$$
\begin{aligned}
& a=\alpha q+q_{1}, 0 \leq q_{1}<q, \text { and } \\
& \gamma q_{1}=q+q_{2}, 0 \leq q_{2}<q_{1}\left(\text { if } \mathrm{q}_{1}>0\right) .
\end{aligned}
$$

Note that $r \geq 2$ if $q_{1}>0$. We need to prove that

$$
\delta_{1}(E) \leq a+p-2 .
$$

Claim 1. One can assume that

i ) $l \leq q-1$,

ii) $n>(\alpha-1) p$, and

iii) $q_{1}>0$.

Proof. i) If $l \geq q$, then $\delta_{1}(E) \leq n+l \leq a-2$, since $E=(l-q)+(n+1) a$ and all the coefficients in this equation are nonnegative.

ii) If $n \leq(\alpha-1) p$, let $n=x p+y$, where $0 \leq x \leq \alpha-1$ and $0 \leq y<p$. Then $E=x d+\left[(\alpha-1-x) q+q_{1}+\eta\right]+y a$. Hence

$$
\begin{aligned}
\delta_{1}(E) & \leq x+(\alpha-1-x) q+q_{1}+l+y \\
& \leq x+(\alpha-1-x) q+q_{1}+q-1+p-1(\text { by }(\mathrm{i})) \\
& \leq \alpha-1+p-1 .
\end{aligned}
$$

iii) Assume that $q_{1}=0$, i.e., $a=\alpha q$. By ii) above we have $n^{\prime}=n-(\alpha-1) p$ $>0$. Then $E=(\alpha-1) q+l+(\alpha-1) p a+n^{\prime} a=(\alpha-1) d+l+n^{\prime} a$. Hence $\delta_{1}(E) \leq l+n^{\prime}+\alpha-1 \leq l+n \leq a-2$. Note that this case also follows easily from Section 3.

We now divide into cases.

Case 1. $n \leq \alpha p+(\gamma-1)(1+\alpha p)-1$.

Case 1a: $\alpha \geq 2$ and $q_{1} \geq 2 p+1$

Looking at the representation

$$
E=(\alpha-1) d+\left(l+q_{1}\right)+[n-(\alpha-1) p] a,
$$


we get

$$
\begin{aligned}
\delta_{1}(E) & \leq q_{1}+l+n-(\alpha-1)(p-1) \\
& \leq q_{1}+q-1+\alpha p+(\gamma-1)(1+\alpha p)-1-(\alpha-1)(p-1) \\
& =a+p-2-\alpha[q-(\gamma-1) p-1]+(\gamma-1)+q-1 .
\end{aligned}
$$

Since $\alpha \geq 2$ it follows easily that it suffices to show that $2 q \geq(2 p+1)(\gamma-1)$ $+q+1$. But this is immediate from the following inequalities: $q-1=\gamma q_{1}-q_{2}$ $-1 \geq(\gamma-1) q_{1} \geq(\gamma-1)(2 p+1)$ (here we use our hypothesis).

Case 1 b: $\alpha=1$, i.e. $a=q+q_{1}$.

Let $i$ be the smallest integer such that

$$
n-i(p+1)+1<0 .
$$

By the assumption of this case and by Claim 1(ii), $0<i \leq \gamma$. Then the coefficient of $a$ in the following equation is nonnegative:

$$
E=(i-1) d+\left[l+i q_{1}-a\right]+[n-(i-1)(p+1)+1] a .
$$

As in the proof of Claim 1(i) from this equation we obtain $\delta_{1}(E) \leq a+p-2$ if $l+i q_{1}-a \geq 0$. Let $l+i q_{1}-a<0$. Using the representation (1)(with $\alpha=1$ ) and the bounds on $n$ and $l$ we get $\delta_{1}(E) \leq l+n+q_{1} \leq a-i q_{1}-1+i(p+1)$ $-2+q_{1}=a+p-2+(i-1)\left(p+1-q_{1}\right) \leq a+p-2 \quad$ (since $a=q+q_{1}$ $\geq p+q+1$ and $i \geq 1$ ).

Case 1c: $\alpha \geq 2$ and $q_{1} \leq 2 p$.

Looking again at (2) it suffices to consider the case $q_{1} \geq(\alpha-1)(p-1)+p$ $+1 \geq 2 p$. Hence by combining with Case 1 a we have still to consider the case $\alpha=2$ and $q_{1}=2 p$. We have

$$
E=2 i d+[l+2 i p-q]+[n+1-i(1+2 p)] a,
$$

for arbitrary $i$. Similarly to Case $1 \mathrm{~b}$, we choose $i$ to be the smallest integer such that

$$
n+1-(i+1)(1+2 p)<0 \text {. }
$$

By Claim 1(ii) and the assumption of our Case $1,0 \leq i<\gamma \leq q$. After using the above equation for $E$ in (3), we have still to consider the following case, namely

$$
l+2 i p-q<0 .
$$

Using the representation $E=1 \cdot d+(l+2 p)+(n-p) a$ and the above bounds 
for $n, l, i$ we get $\delta_{1}(E) \leq n+l+p+1 \leq(i+1)(1+2 p)-2+q-2 i p-$ $1+p+1=a+p-2-q+i+1 \leq a+p+2$ (since $i<q$ ). This completes the proof of Case 1 .

Case 2. $n \geq \alpha p+(\gamma-1)(1+\alpha p)$.

We have

$$
\begin{aligned}
E= & (\alpha-1) d+\left(l+q_{1}\right)+[n-(\alpha-1) p] a \\
= & (\alpha-1) d+l+q_{1}+\alpha q+q_{1}+[n-(\alpha-1) p-1] a \\
= & {[\alpha-1+\alpha] d+\left(l+2 q_{1}\right)+[n-(\alpha-1) p-(1+\alpha p)] a } \\
& \cdots \ldots \\
= & {[(\alpha-1)+(\gamma-2) \alpha] d+\left[l+(\gamma-1) q_{1}\right]+} \\
& \quad[n-(\alpha-1) p-(\gamma-2)(1+\alpha p)] a \\
= & {[\alpha+(\gamma-2) \alpha] d+\left[l-\left(q_{1}-q_{2}\right)\right]+[n-\alpha p-(\gamma-2)(1+\alpha p)] a . }
\end{aligned}
$$

Looking at the last expression of $E$, similarly as in the proof of Claim 1(i), we get

Claim 2. One can assume that $l<q_{1}-q_{2}$.

Next we define a new sequence of representations of $E$. Continuing the above procedure, we get the following expression of $E$ :

$$
E=[\alpha+(\gamma-1) \alpha] d+\left(l+q_{2}\right)+[n-\alpha p-(\gamma-1)(1+\alpha p)] a .
$$

We set $A_{1}=\alpha+(\gamma-1) \alpha, B_{1}=q_{2}$ and $C_{1}=n-\alpha p-(\gamma-1)(1+\alpha p)$. Assume that we have found an $i$-th expression:

$$
E=A_{i} d+l+B_{i}+C_{i} a .
$$

We use again the above procedure in order to find a new one.

If $l+B_{i}-\left(q_{1}-q_{2}\right) \geq 0$ then the $(i+1)$-st expression of $E$, obtained by iterating

$$
\begin{aligned}
E & =A_{i} d+l+B_{i}+C_{i} a=A_{i} d+l+B_{i}+\alpha q+q_{1}+\left(C_{i}-1\right) a \\
& =\left(A_{i}+\alpha\right) d+\left(l+B_{i}+q_{1}\right)+\left(C_{i}-1-\alpha p\right) a=\cdots,
\end{aligned}
$$

is

$$
\begin{aligned}
E & =\left[A_{i}+(\gamma-1) \alpha\right] d+l+B_{i}+(\gamma-1) q_{1}+\left[C_{i}-(\gamma-1)(1+\alpha p)\right] a \\
& =\left[A_{i}+1+(\gamma-1) \alpha\right] d+\left[l+B_{i}-\left(q_{1}-q_{2}\right)\right]+\left[C_{1}-p-(\gamma-1)(1+\alpha p)\right] a .
\end{aligned}
$$

That means, in this case we set $A_{i+1}=A_{i}+1+(\gamma-1) \alpha, B_{i+1}=B_{i}-\left(q_{1}-\right.$ $\left.q_{2}\right)$ and $C_{i+1}=C_{i}-p-(\gamma-1)(1+\alpha p)$. 
If $l+B_{i}<q_{1}-q_{2}$ then we consider the following $(i+1)$-st expression of $E$ :

$$
\begin{aligned}
E & =\left[A_{i}+\gamma \alpha\right] d+l+B_{\imath}+\gamma q_{1}+\left[C_{i}-\gamma(1+\alpha p)\right] a \\
& =\left[A_{i}+1+\gamma \alpha\right] d+\left[l+B_{\imath}+q_{2}\right]+\left[C_{i}-p-\gamma(1+\alpha p)\right] a .
\end{aligned}
$$

So, in this case we set $A_{i+1}=A_{i}+1+\gamma \alpha, B_{i+1}=B_{i}+q_{2}$ and $C_{\imath+1}=C_{i}-p-$ $\gamma(1+\alpha p)$.

From the above procedure and by Claim 2 we immediately get:

Claim 3. For all $i \geq 1$ we have $E=A_{i} d+l+B_{i}+C_{i} a$ with $A_{i}, B_{i}, C_{i}$ being defined as follows:

i)

$$
\begin{aligned}
& A_{i}=\alpha-1+i+\left(\beta_{1}+\cdots+\beta_{i}\right) \alpha, \text { and } \\
& C_{i}=n-(\alpha-1+i) p-\left(\beta_{1}+\cdots+\beta_{i}\right)(1+\alpha p),
\end{aligned}
$$

where $\beta_{1}=\gamma-1$, and for $i \geq 1$

$$
\beta_{i+1}= \begin{cases}\gamma & \text { if } l+B_{i}<q_{1}-q_{2}, \\ \gamma-1 & \text { otherwise. }\end{cases}
$$

ii) $B_{1}=q_{2}$ and

$$
\beta_{i+1}= \begin{cases}B_{i}+q_{2} & \text { if } B_{i+1}=\gamma, \\ B_{i}-\left(q_{1}-q_{2}\right) & \text { if } B_{i+1}=\gamma-1 .\end{cases}
$$

iii) $0 \leq l+B_{i}<q_{1}$.

Let $s$ be the smallest integer such that $C_{s+1}<0$. By the hypothesis of Case 2, $s \geq 1$. Then all above expressions of $E$ with $i \leq s$ are indeed representations of $E$. Hence for $1 \leq i \leq s$ we get from Claim 3 (i) that

(4) $\delta_{1}(E) \leq l+B_{i}+A_{i}+C_{i}$

$$
=B_{i}+l+n-(\alpha-1+i)(p-1)-\left(\beta_{1}+\cdots+\beta_{i}\right)(1+\alpha p-\alpha) \text {. }
$$

Since $l+n \leq a-2$, we conclude (otherwise there is nothing left to prove):

Claim 4. One can assume that for $1 \leq i \leq s$,

$$
B_{i} \geq(\alpha-1+i)(p-1)+\left(\beta_{1}+\cdots+\beta_{\imath}\right)(1+\alpha p-\alpha)+p+1 .
$$

We now consider two subcases. 
Case 2a: $\left(p, \gamma, \beta_{s+1}\right) \neq(1,2,1)$.

For short, let $\beta=\beta_{1}+\cdots+\beta_{s}$. The condition $C_{s+1}<0$ means that

$$
n \leq(\alpha+s) p+\left(\beta+\beta_{s+1}\right)(1+\alpha p)-1 .
$$

Hence, from (4) with $i=s$ and Claim 3 (iii) we get

$$
\begin{aligned}
\delta_{1}(E) \leq & \left(l+B_{s}\right)+n-(\alpha-1+s)(p-1)-\beta(1+\alpha p-\alpha) \\
\leq & q_{1}-1+(\alpha+s) p+\left(\beta+\beta_{s+1}\right)(1+\alpha p)-1 \\
& -(\alpha-1+s)(p-1)-\beta(1+\alpha p-\alpha) \\
= & q_{1}+p-2+\alpha+s+\beta_{s+1}(1+\alpha p)+\beta \alpha-1 \\
= & a+p-2-\alpha q+\alpha+s+\beta_{s+1}(1+\alpha p)+\beta \alpha-1 .
\end{aligned}
$$

We need to show that $\alpha q \geq \alpha+s+\beta_{s+1}(1+\alpha p)+\beta \alpha-1$, or, equivalently,

$$
\alpha\left(q-1-p \beta_{s+1}-\beta\right) \geq \beta_{s+1}+s-1 \text {. }
$$

If $\beta_{s+1}=\gamma$ then, by Claim 3 (i), $B_{s}<q_{1}-q_{2}$. Hence, by Claim 4 , we have

$$
\begin{aligned}
q & =\gamma q_{1}-q_{2} \geq \gamma\left(q_{1}-q_{2}\right) \geq \gamma\left(B_{s}+1\right) \\
& \geq \gamma[(\alpha-1+s)(p-1)+\beta(1+\alpha p-\alpha)+p+2] \\
& \geq \gamma(\beta p+p+2) \\
& \geq \gamma(p+1)+1+2 \beta \text { (because } \gamma \geq 2) .
\end{aligned}
$$

Since $\beta=\beta_{1}+\cdots+\beta_{s} \geq s$, we then get

$$
\begin{aligned}
\alpha\left(q-1-p \beta_{s+1}-\beta\right) & =\alpha(q-1-p \gamma-\beta) \geq q-1-\gamma p-\beta \\
& \geq \gamma+\beta=\beta_{s+1}+\beta \geq \beta_{s+1}+s>\beta_{s+1}+s-1,
\end{aligned}
$$

which gives (6).

If $\beta_{s+1}=\gamma-1$, then by Claim 3 (iii) and Claim 4 we have

$$
\begin{aligned}
q-1 & =\gamma q_{1}-q_{2}-1 \geq(\gamma-1) q_{1} \geq(\gamma-1)\left(B_{s}+1\right) \\
& \geq(\gamma-1)[(\alpha-1+s)(p-1)+\beta(1+\alpha p-\alpha)+p+2] \\
& \geq(p+1)(\gamma-1)+1+s(p-1)+(\gamma-2) \beta+\beta \\
& \geq \beta+(p+1)(\gamma-1)+s(p-1+\gamma-2) \text { (because } \beta \geq s) . \\
& =\beta+(p+1) \beta_{s+1}+s(p-1+\gamma-2) .
\end{aligned}
$$

From this it follows, since $p>1$ or $\gamma>2$, that (6) holds.

Case $2 \mathrm{~b}: p=1, \gamma=2$ and $\beta_{s+1}=1$.

If $\beta_{1}=\cdots=\beta_{s+1}=1$, then by Claim 3 (ii) $B_{s}=B_{1}-(s-1)\left(q_{1}-q_{2}\right)=$ $q_{2}-(s-1)\left(q_{1}-q_{2}\right) \leq q_{1}-s$. Hence, by Claim 4 , we get $q>q_{1} \geq s+B_{s} \geq s$ $+\beta+2$, where $\beta$ is defined as in Case $2 \mathrm{a}$. This shows the inequality (6) for 
$p=1, \beta_{s+1}=1$ and we are done.

Finally, assume that not all $\beta_{i}$ are equal to 1 . Let $j$ be the largest integer such that $\beta_{j+1}=2$. Thus $1 \leq j \leq s-1$ and $\beta_{j+2}=\cdots=\beta_{s+1}=1$. By Claim 3 (i), $l+B_{j}<q_{1}-q_{2}$. By Claim 4 it then implies that $q_{1}-q_{2}>2$. Using (4) with $i=j$ and (5) we get:

$$
\begin{aligned}
\delta_{1}(E) & \leq q_{1}-q_{2}-1+(s+\alpha)+(\alpha+1)\left(\beta_{1}+\cdots+\beta_{j}+\cdots+B_{s+1}\right)-1- \\
& \left(\beta_{1}+\cdots+\beta_{j}\right) \\
& =q_{1}-q_{2}-1+(\alpha+s)+\alpha\left(\beta_{1}+\cdots+\beta_{j}\right)-1+(\alpha+1)(s+2-j) \\
& =a-1-\alpha q-q_{2}+\alpha\left(\beta_{1}+\cdots+\beta_{j}\right)+\alpha(s+3-j)+2 s+1-j .
\end{aligned}
$$

We need to show that

$$
\alpha\left[q-\left(\beta_{1}+\cdots+\beta_{j}\right)-(s+3-j)\right]+q_{2} \geq 2 s+1-j .
$$

Since $\beta_{j+2}=\cdots=\beta_{s+1}=1(=\gamma-1)$, Claim 3 (ii) and Claim 4 give us that

$$
B_{s}=B_{j+1}-(s-j-1)\left(q_{1}-q_{2}\right) \geq \beta_{1}+\cdots+\beta_{s}+2 .
$$

Hence, by Claim 3 (iii),

$$
\begin{aligned}
q_{1} & \geq B_{j+1}+1 \geq(s-j-1)\left(q_{1}-q_{2}\right)+\beta_{1}+\cdots+\beta_{s}+3 \\
& \geq 2(s-j-1)+s+3=3 s-2 j+1 .
\end{aligned}
$$

So,

(9) $2 q_{1} \geq 3 s-2 j+1+\beta_{1}+\cdots+\beta_{j}+3=\beta_{1}+\cdots+\beta_{j}+3 s-2 j+4$.

Since $q \geq q_{1}+1$ and $q_{1}-q_{2} \geq 1$, from (8) it follows that $q \geq\left(\beta_{1}+\cdots+\beta_{s}\right)$ $+s+3-j \geq\left(\beta_{1}+\cdots+\beta_{j}\right)+(s+3-j)$. That means $q-\left(\beta_{1}+\cdots+\beta_{j}\right)$ $-(s+3-j) \geq 0$. Hence

$$
\begin{aligned}
\alpha\left[q-\left(\beta_{1}+\cdots+\beta_{j}\right)\right. & -(s+3-j)]+q_{2} \geq q-\left(\beta_{1}+\cdots+\beta_{j}\right)- \\
& (s+3-j)+q_{2} \\
& =2 q_{1}-\left(\beta_{1}+\cdots+\beta_{j}\right)-(s+3-j)(\text { since } \gamma=2) \\
& \geq 2 s+1-j \quad(\text { by }(9)),
\end{aligned}
$$

which shows (7). This completes the proof of Case $2 \mathrm{~b}$ and therefore the proof of Lemma 5.2 .

\section{Liaison among monomial curves in $P_{K}^{3}$}

In $[\mathrm{BH}]$ liaison amongst monomial curves in $P_{K}^{3}$ is investigated. There it is shown that some linkage classes contain only few monomial curves, while in other 
linkage classes there are infinitely many monomial curves. Recall that two curves $C, C^{\prime}$ of $P^{3}$ are said to be linked if there is an $R$-regular sequence $\{f, g\} \in$ $I(C) \cap I\left(C^{\prime}\right)$ such that $I(C)=(f, g): I\left(C^{\prime}\right)$ and $I\left(C^{\prime}\right)=(f, g): I(C)$. In this case one writes $C \sim C^{\prime} . C$ and $C^{\prime}$ are in the same linkage class (the same even linkage class) if there exists a sequence of links $C=C_{0} \sim C_{1} \sim \cdots \sim C_{m}=C^{\prime}$ (with $m$ even, resp.).

Using the notion of $k$-Buchsbaum curves we can get some new insight on liaison among monomial curves. An easy consequence of $[R]$ is that two curves in the same linkage class have the same Buchsbaum nunber (see $[\mathrm{FH}]$, Section $5 \mathrm{~b}$ ). For $k \leq 2$, one can define all linkage classes of strictly $k$-Buchsbaum monomial curves (see $[\mathrm{BSV}]$ and $[\mathrm{H}]$ ). It is natural to ask whether for a given $k$ there are only finitely many linkage classes among strictly $k$-Buchsbaum monomial curves. Using Theorem 2.1 we answer it in the affirmative.

Proposition 6.1. Assume $C\left(n_{1}, n_{2}, n_{3}\right)$ is $k$-Buchsbaum for a nonnegative integer $k$. Then

(i) The number of $S^{\prime}$-minimal elements in $S^{\prime} \backslash S$ is $k$.

(ii) $\mu\left(I\left(C\left(n_{1}, n_{2}, n_{3}\right)\right)\right) \leq \begin{cases}3 & \text { if } k=0 \\ 2 k+2 & \text { if } k \geq 1\end{cases}$

Proof. By Theorem 2.1 we have $H_{\underline{m}}^{1}(K[S])=M_{a+1} \oplus \cdots \oplus M_{a+k}$, where $a\left(H_{\underline{m}}^{1}(K[S])\right)=a+1$. Assume $k \geq 1$ (the case $k=0$ being true vacuously for (i)). Let, in the notation of Section $3, \frac{x_{2}^{a_{2}}}{x_{3}^{a_{3}}} \in H_{\underline{m}}^{1}(\bar{R})$ correspond to the first element in $\mathscr{B}_{2}$ (recall that $\left.\bar{R} \cong K[S]\right)$. Then $d\left(\frac{x_{2}^{a_{2}}}{x_{3}^{a_{3}}}\right) \geq a+1$. As in Section $3,0 \neq$ $x_{0}^{\alpha_{0}-1} x_{3}^{\alpha_{3}-1} \frac{x_{2}^{a_{2}}}{x_{3}^{a_{3}}} x_{1}^{b_{1}-1} x_{2}^{b_{2}-1} \in H_{\underline{m}}^{1}(\bar{R})$. Hence $a_{0}-1+a+1 \leq d\left(x_{0}^{\alpha_{0}-1} \frac{x_{2}^{a_{2}}}{x_{3}^{a_{3}}}\right) \leq a+k$, from which $a_{0} \leq k$. From this we have immediately $\left|\mathscr{B}_{2}\right| \leq k$, since the $x_{0}$-exponent decreases by at least one in every step of the algorithm in [BR], which proves (i). If $k=0$, then (ii) follows from [B2]. Assume $k \geq 1$. Then $\mathscr{B}_{3} \neq \emptyset$ with $x_{0}^{b_{0}} x_{3}^{b_{3}}-x_{1}^{b_{1}} x_{2}^{b_{2}}$ as its first element. As before we have $b_{1}-1+a+1 \leq a+k$ or $b_{1} \leq k$, from which $\left|\mathscr{B}_{3}\right| \leq k$, since the $x_{1}$-exponent decreases by at least one in every step of the algorithm in [BR]. Thus $\mu\left(I\left(C\left(n_{1}, n_{2}, n_{3}\right)\right)\right) \leq 2 k+2$, which proves (ii).

Remark 6.1. The upper bound in (i) and (ii) is sharp, it is attained already 
for $k=0,1,2$ (see [B2], [BSV] and [H]).

THEOREM 6.1. Let $k$ be a fixed nonnegative integer. Then there are only finitely many linkage classes for strictly $k$-Buchsbaum monomial curves in $P_{K}^{3}$.

Proof. Since $k=\operatorname{diam} M(C)$, by Theorem $2.1 M(C)$ has the following form:

$$
M(C) \cong H_{\underline{m}}^{1}(K[S]) \cong k\left[S^{\prime} \backslash S\right]=M_{a+1} \oplus \cdots \oplus M_{a+k}
$$

We may consider modules over $K\left[x_{0}, x_{1}, x_{2}, x_{3}\right]=R$ or $K[S]$ via the ring homomorphism: $x_{0} \mapsto s^{n_{3}}, x_{1} \mapsto s^{n_{3}-n_{1}} t^{n_{1}}, x_{2} \mapsto s^{n_{3}-n_{2}} t^{n_{2}}, x_{3} \mapsto t^{n_{3}}$. Since the HartshorneRao module $M(C)$ is invariant under linkage (up to $\mathrm{K}$-duality and a shift in grading), we have $M(C) \cong M\left(C^{\prime}\right)(n)$ or $M(C) \cong \operatorname{Ext}_{R}^{4}\left(M\left(C^{\prime}\right), R\right)(-n)$. Thus it is enough to show that there are only finitely many different possible $R$-modules of the above form. This is equivalent to showing that the dimension $\left|M_{i}\right|$ of $K$-vector space $M_{i}$, is finite for all $a+1 \leq i \leq a+k$. But an element of $S^{\prime} \backslash S$ of degree $i$ is of the form $f_{1}+\cdots+f_{g}$ (with possible repetition), where $f_{j}$ is an $S^{\prime}$-minimal element of $S^{\prime} \backslash S$ or $f_{j} \in\left\{t_{0}, t_{1}, t_{2}, t_{3}\right\}$, and $1 \leq g \leq i-a$. Hence, by Proposition 6.1, $\left|M_{i}\right| \leq(k+4)^{i-a} \leq(k+4)^{k}$, as required.

In $[\mathrm{BH}]$ we have an algorithm for determining whether two monomial curves are in the same even linkage class. Here we give a particular result in the "opposite" direction.

Proposition 6.2. No two non $C-M$ curves of the type $C(1, a, d)$ are in the same even linkage class.

Proof. Assume that $C=C(1, a, d)$ and $C^{*}=C\left(1, a^{*}, d^{*}\right)$ are in the same even linkage class. Then $k(C)=k\left(C^{*}\right)=k>0$. By Theorem 5.1 we get $a=a^{*}$ $=k+2$. One needs to show that $d=d^{*}$. Let $d=p a+q$ and $d^{*}=p^{*} a+q^{*}$, where $p, q, p^{*}, q^{*}$ are positive integers such that $p+q \leq a-1$ and $p^{*}+q^{*}$ $\leq a-1$ (by Example 4.1 and our assumption that $C$ and $C^{*}$ are not $\mathrm{C}-\mathrm{M}$ ). Consider the element $e=((p+1)(d-a), a-q)$. By Lemma 5.1 it follows that $e$ has the minimal degree (which equals to $p$ ) among the elements of $S^{\prime} \backslash S$. Moreover it is unique with this property if $p>1$. If $p=1$ there are maybe some elements of $S^{\prime} \backslash S$ having the minimal degree. But $e$ is still the unique element in $S^{\prime} \backslash S$ which satisfies the following two properties:

i ) $\delta(e)$ is minimal among $\delta\left(e^{\prime}\right), e^{\prime} \in S^{\prime} \backslash S$,

ii) $e+t_{3} \in S$.

The last property is true for any $p$. For the $R$-module $M(C)$ these properties 
mean that:

$i^{\prime}$ ) $e$ has the minimal degree among homogeneous generators of $M(C)$ (we identify $e$ with the corresponding element in $\left.M(C) \cong H_{m}^{1}(K[S]) \cong K\left[S^{\prime} \backslash S\right]\right)$,

ii') $x_{3} \cdot e=0$.

Hence, for any curve $C$ there is unique generator $e$ defined by the above two properties (up to a scalar). For $C^{*}$, let $e^{*}=\left(\left(p^{*}+1\right)\left(d^{*}-a\right), a-q^{*}\right) \in\left(S^{*}\right)^{\prime} \backslash S^{*}$. From the above observation and by $[\mathrm{R}], M(C) \cong M\left(C^{*}\right)(n)$, we deduce that $e \mapsto e^{*}$.

By Lemma 2.1 it follows that $e+q t_{1} \in S$, but $e+(q-1) t_{1} \in S^{\prime} \backslash S$ (and an analogous statement for $e^{*}$ and $q^{*}$ ). This means that $q$ (resp. $q^{*}$ ) is the smallest integer such that $x_{1}^{q} e=0$ in $M(C)\left(x_{1}^{q^{*}} e^{*}=0\right.$ in $M\left(C^{*}\right)$, resp.). Hence $q=q^{*}$. Analogously, $m=a-p-q$ (resp. $m^{*}=a-p^{*}-q$ ) is the smallest integer such that $x_{0}^{m} e=0$ in $M(C)$ (resp. $x_{0}^{m^{*}} e^{*}=0$ in $M\left(C^{*}\right)$, resp.). Therefore $a-p$ $-q=a-p^{*}-q$, so $p=p^{*}$, as required.

The following corollary examines even linkage between curves $C=C(1, a, d)$ and $C^{*}=C\left(d^{*}-a^{*}, d^{*}-1, d^{*}\right)$.

Corollary 6.1. Let $C(1, a, b)$ be a not $C-M$ curve such that $d>a+1$. If $C$ and $C^{*}=C\left(d^{*}-a^{*}, d^{*}-1, d^{*}\right)$ are in the same even linkage class, then $a=$ $a^{*}=p+q+1=p^{*}+q^{*}+1$, where $d=p a+q, 0<q<a$, and $d^{*}=p^{*} a^{*}$ $+q^{*}, 0<q^{*}<a^{*}$.

Proof. By Theorem 5.1, $a-2=k(C)=k\left(C^{*}\right)=a^{*}-2$, thus $a=a^{*}$. To prove $p+q+1=a=a^{*}=p^{*}+q^{*}+1$ let $S^{*}$ denote the associated semigroup of $C^{*}$. As in the proof of Proposition 6.2, there is a uniquely defined element $f \in\left(S^{*}\right)^{\prime} \backslash S^{*}$ such that $f$ has the minimal degree and $x_{0} f=0$. (Note that for $I\left(C^{*}\right)$ one needs to permute the variables $x_{0} \leftrightarrow x_{3}, x_{1} \leftrightarrow x_{2}$.) Let $e \in S^{\prime} \backslash S$ be the image of $f$ in the isomorphism $M(C) \cong M\left(C^{*}\right)(n)$. Then $e \in\left[S^{\prime} \backslash S\right]_{p}$, the $p$-degree component, and $x_{0} e=0$. If $\left[S^{\prime} \backslash S\right]_{p}$ has only one element, then by the proof of Proposition 6.2, the smallest integer $m$ such that $x_{0}^{m} e=0 \in M(C)$ is $a-p-q$. Thus $1=a-p-q$. By symmetry $a^{*}=p^{*}+q^{*}+1=a$. We will show next, that the above is the only possible case. Suppose therefore that $\left[S^{\prime} \backslash S\right]_{p}$ and $\left[\left(S^{*}\right)^{\prime} \backslash\left(S^{*}\right)\right]_{p^{*}}$ have at least two elements. Then, by Lemma 5.1 (or by the algorithm [BR]), $p=p^{*}=1$. Since $x_{0} e=0$, i.e. $t_{0}+e \in S$, from Lemma 5.1 and Lemma 2.1 we get that $e=((1+\beta)(d-a), a-\beta q)$ and $a-\beta q=2$. Note that $C\left(1, a, d^{*}\right)$ and $C(d-a, d-1, d)$ are also in the same even linkage class. Hence, as before, $a-\beta^{*} q^{*}=2$. Again by Lemma $5.1, \beta$ and $\beta^{*}$ equal the 
number of elements in $\left[S^{\prime} \backslash S\right]_{1}$ and $\left[\left(S^{*}\right)^{\prime} \backslash\left(S^{*}\right)\right]_{1}$, respectively. Therefore $\beta=$ $\beta^{*} \geq 2$, which implies $q=q^{*}$. By the proof of Proposition $6.2, x_{3}^{a-p^{*}-q^{*}-1} f \neq 0$, hence $x_{3}^{a-q-2} e \neq 0$, i.e. $(a-q-2) t_{3}+e \notin S$. By Lemma 2.1 this implies $\beta+1$ $>a-q-1$, or equivalently $0 \geq(\beta-1)(q-1)$ (since $a=\beta q+2)$. But this is impossible since $\beta \geq 2$ and $q>1$ by our initial assumption $d>a+1$. This contradiction finishes the proof.

Remark 6.2. (i) An easy example shows that it is possible to have $d=d^{*}$ or $d \neq d^{*}$ and $C(1, a, d)$ and $C\left(d^{*}-a^{*}, d^{*}-1, d^{*}\right)$ be in the same even linkage class.

(ii) It is shown in [BSV] and [H] that for $k \leq 2$ each linkage class of strictly $k$-Buchsbaum monomial curves contains a curve $C(1, a, d)$. That this is not true for all nonnegative integers $k$ was shown by M. Morales in [Mo]. We therefore conclude our paper with the following open problem: Determine all linkage classes of monomial curves in $P_{K}^{3}$ which contain a representative $C(1, a, d)$ or $C(d-a$, $d-1, d)$ and determine the integer $a$ for these linkage classes.

\section{REFERENCES}

[B1] H. Bresinsky, Monomial Buchsbaum ideals in $P^{r}$, manuscripta Math., 47 (1984), $105-132$.

[B2] - Minimal free resolutions of monomial curves in $P_{K}^{3}$, Linear Alg. and Appl., 59 (1984), 121-129.

[BH] H. Bresinsky and C. Huneke, Liaison of monomial curves in $P^{3}$, J. reine angew. Math., 365 (1986), 33-66.

[BR] H. Bresinsky and B. Renschuch, Basisbestimmung Veronesescher Projektionsideale mit allgemeiner Nullstelle $\left(t_{0}^{m}, t_{0}^{m-r} t_{1}^{r}, t_{0}^{m-s} t_{1}^{s}, t_{1}^{m}\right)$, Math. Nachr., 96 (1980), $257-269$.

[BSV] H. Bresinsky, P. Schenzel and W. Vogel, On liaison, arithmetically Buchsbaum curves and monomial curves in $P^{3}$, J. Algebra, 86 (1984), 283-301.

[BSS] H. Bresinsky, U. Schäfer and P. Schenzel, On the canonical module for monomial curves, Comm. in Algebra, 15 (1987), 1799-1814.

[EG] D. Eisenbud and S. Goto, Linear free resolutions and minimal multiplicity, J. Algebra, 88 (1984), 89-133.

[FH] M. Fiorentini and L. T. Hoa, On monomial $k$-Buchsbaum curves in $P^{n}$, Ann. Univ. Ferrara, Sez. VII, Sci. Mat., 36 (1990), 159-174.

[FV] M. Fiorentini and W. Vogel, Old and new results and problems on Buchsbaum rings, I. Seminari di Geometria 1988-1991, pp. 53-61 Univ. Bologna 1991.

[GMV] A. V. Geramita, P. Maroscia and W. Vogel, A note on arithmetically Buchsbaum curves in $P^{3}$, Matematiche (Catania), 40 (1985), 21-28.

[GW] A. V. Geramita and C. A. Weibel, On the Cohen-Macaulay and Buchsbaum property for unions of planes in $A^{n+1}(k)$, J. Algebra, 92 (1985), 413-445.

[H] L. T. Hoa, On monomial $k$-Buchsbaum curves in $P^{3}$, Manuscripta Math., 73 
(1991), 423-436.

[HV] L. T. Hoa and W. Vogel, Castelnuovo-Mumford regularity and hyperplane sections, J. Algebra (to appear).

[K] J. Kästner, $\mathrm{Zu}$ einem Problem von H. Bresinsky über monomiale Buchsbaum Kurven, Manuscripta Math., 54 (1985), 197-204.

[MM] J. C. Migliore and R. M. Miro-Roig, On $k$-Buchsbaum curves in $P^{3}$, Comm. in Algebra, 18 (1990), 2403-2422.

[Mo] M. Morales, Courbes monomiales dans l'espace projectif, module de Rao et liaison, Preprint Institute Fourier (Grenoble) 1993.

[M] D. Mumford, Lectures on curves on an algebraic surface, Ann. of Math. Studies 59, Princeton Univ. Press., Princeton, N. J., 1966.

[R] A. P. Rao, Liaison among curves in $P_{K}^{3}$, Invent. Math., 50 (1979), 205-217.

[Ro] O. J. Rödseth, On a linear diophantine problem of Frobenius, J. reine angew. Math., 301 (1978), 171-178.

[SV] J. Stückrad and W. Vogel, Buchsbaum rings and applications, Springer Verlag 1986

[TH] N. V. Trung and L. T. Hoa, Affine semigroups and Cohen-Macaulay rings generated by monomials, Trans. Amer. Math. Soc., 298 (1986), 145-167.

\author{
Henrik Bresinsky \\ Frank Curtis \\ 414 and 336 Neville Hall \\ University of Maine \\ Orono, Maine 04469-5752 (U.S.A.) \\ Mario Fiorentini \\ Dipartimento di Matematica \\ Universita di Ferrara \\ Via Machiavelli 35 \\ Ferrara 44100 (Italy) \\ Lê Tuân Hoa \\ Institute of Mathematics \\ P. O. Box 631, Bo ho, \\ Hanoi (Vietnam)
}

